\title{
Statistical analysis of intrinsic polarization, IR excess and projected rotational velocity distributions of classical Be stars ${ }^{\star}$
}

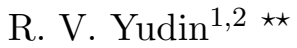 \\ 1 Central Astronomical Observatory of the Russian Academy of Sciences at Pulkovo, 196140 Saint-Petersburg, \\ Russia \\ 2 Isaac Newton Institute of Chile, St.-Petersburg Branch, Chile
}

Received 9 December 1999 / Accepted 22 December 2000

\begin{abstract}
We present the results of statistical analyses of a sample of 627 Be stars. The parameters of intrinsic polarization $\left(p_{*}\right)$, projected rotational velocity $(v \sin i)$, and near IR excesses have been investigated. The values of $p_{*}$ have been estimated for a much larger and more representative sample of Be stars $(\approx 490$ objects $)$ than previously. We have confirmed that most Be stars of early spectral type have statistically larger values of polarization and IR excesses in comparison with the late spectral type stars. It is found that the distributions of $p_{*}$ diverge considerably for the different spectral subgroups. In contrast to late spectral types (B5-B9.5), the distribution of $p_{*}$ for B0-B2 stars does not peak at the value $p_{*}=0 \%$. Statistically significant differences in the mean projected rotational velocities $(\overline{v \sin i})$ are found for different spectral subgroups of Be stars in the sense that late spectral type stars (V luminosity class) generally rotate faster than early types, in agreement with previously published results. This behaviour is, however, not obvious for the III-IV luminosity class stars. Nevertheless, the calculated values of the ratio $v_{\mathrm{t}} / v_{\mathrm{c}}$ of the true rotational velocity, $v_{\mathrm{t}}$, to the critical velocity for break-up, $v_{\mathrm{c}}$, is larger for late spectral type stars of all luminosity classes. Thus, late spectral type stars appear to rotate closer to their break-up rotational velocity. The distribution of near IR excesses for early spectral subgroups is bi-modal, the position of the second peak displaying a maximum value $E(V-L) \approx 1.3$ for $\mathrm{O}-\mathrm{B} 1.5$ stars, decreasing to $E(V-L) \approx 0$ m. 8 for intermediate spectral types (B3-B5). It is shown that bi-modality disappears for late spectral types (B6-B9.5). No correlations were found between $p_{*}$ and near IR excesses and between $E(V-L)$ and $v \sin i$ for the different subgroups of Be stars. In contrast to near IR excesses, a relation between $p_{*}$ and far IR excesses at $12 \mu \mathrm{m}$ is clearly seen. A clear relation between $p_{*}$ and $v \sin i$ (as well as between $p_{*}$ and $\overline{v \sin i} / v_{\mathrm{c}}$ ) is found by the fact that plots of these parameters are bounded by a "triangular" distribution of $p_{*}: v \sin i$, with a decrease of $p_{*}$ towards very small and very large $v \sin i$ (and $\overline{v \sin i} / v_{\mathrm{c}}$ ) values. The latter behaviour can be understood in the context of a larger oblateness of circumstellar disks for the stars with a rapid rotation. From the analysis of correlations between different observational parameters we conclude that circumstellar envelopes for the majority of Be stars are optically thin disks with the range of the half-opening angle of $10^{\circ}<\Theta<40^{\circ}$.
\end{abstract}

Key words. classical Be stars: polarization - projected rotational velocities - near IR excesses - far IR excesses

\section{Introduction}

One of the challenging questions in Be stars investigations is the geometrical form of their circumstellar (CS) envelopes. There is still active debate as to the average value of the opening angle of CS disks around classical Be stars and on the application of the wind-compressed disk model (WCD). The ideas on this matter at present are rather controversial. Most authors have considered geometrically thin CS disks with half-opening angles $\Theta<3^{\circ}$ as follows from the WCD theory of Bjorkman \& Cassinelli

\footnotetext{
* Appendices I and II are only available in electronic form at http://www . edpsciences.org

$\star \star$ e-mail: ruslan@pulkovo.spb.su
}

(1993) and Owocki et al. (1994) (see for example Wood et al. 1997; Quirrenbach et al. 1997 etc.). However, acceptance of such a narrow disks faces several problems in the interpretation of spectroscopic characteristics (Moujtahid et al. 1999; Rivinius et al. 1999) and the observed IR excesses in Be stars (Porter 1997). Moreover, the observed phase transitions $-\mathrm{Be} \Leftrightarrow$ Be-shell $\Leftrightarrow$ normal B - cannot be explained in the framework of a geometrically thin disk model (Moujtahid et al. 1999). Others used disk models with larger values of $\Theta \approx 6^{\circ}$ (Porter 1996) or $\Theta \approx 13^{\circ} \rightarrow 15^{\circ}$ (Hanuschik 1996; Waters et al. 1987) removing some of the difficulties. Finally, some authors recently returned to the model of flattened ellipsoids or spheroidal envelopes (Moujtahid et al. 1999) similar 
to those proposed earlier by Doazan \& Thomas (1982). Although a low level of intrinsic polarization of Be stars is considered as an indication that the envelope must be strongly flattened, other geometries can produce the same level of polarization (see Wood et al. 1996a, 1996b). The IR excesses and spectroscopic characteristics, considered separately, also do not provide unique interpretation on CS geometries. Nevertheless, it is well established that the observed IR excess and polarization of radiation for classical Be stars both have a common origin. The excess is due to free-free and free-bound emission from the dense ionized CS gas, with the polarization being engendered by scattering within clouds of free electrons around the star. Moreover, classical Be stars are very fast rotators and at least a few of them rotate close to their break-up velocities. This rapid rotation is considered as a trigger mechanism for CS envelope formation. To obtain some conclusions on the geometry of CS envelopes around classical Be stars, analysis of correlations between different observational parameters seems strongly desirable (see, for example, Coté $\&$ Waters 1987). For the same reason, it is very important to investigate each of the observed parameters statistically for as large a representative sample as possible. During the last 20 years there have been several attempts to carry out such kinds of investigation for large samples of classical Be stars (from 50 up to 200 objects). However, such sample sizes are still sometimes insufficiently large to provide irrefutable conclusions and some authors indicate this fact themselves (see, for example, Quirrenbach et al. 1997 or Ghosh et al. 1999). By taking a relatively large sample of 90 objects, by adding the "new" data from Ghosh et al. (1999) and McDavid $(1999,2000)$ to the "old" data from McLean \& Brown (1978) and Poeckert et al. (1979), Yudin (2000) came to quite different conclusions on the dependence between $p_{*}$ and $v \sin i$ than those of McLean \& Brown (1978) who used only 67 stars.

The general aim of the proposed exercises here is to investigate statistically the data of $p_{*}$ and $v \sin i$ for the largest possible sample of classical Be stars, to compare the behaviour with near and far IR excesses and to search for possible correlations between the parameters. Another aim is to investigate possible differences between spectral subgroups from the general assembly of classical Be stars. These analyses may give new insight on the configuration of CS envelopes.

\section{Selection procedure}

Up till now, there is no statistical information on whether or not classical Be stars can be treated as a homogeneous group. The largest database, SIMBAD, contains only 82 objects which are classified as Be stars but, at the same time, 884 objects are classified as $\mathrm{O}-\mathrm{B}$ emission line stars. The largest revised catalogue of Be stars of Jaschek \& Egret (1982) contains 1155 objects but a significant fraction of these belong to the young Herbig Be star group.
For the statistical analysis here, data for 627 Be stars have been compiled from different sources, taking into account an overlap of the list of emission line stars from the SIMBAD database and the catalogue of Be stars of Jaschek \& Egret (1982). All Be stars which have been considered in the past as young Herbig Be stars have been excluded; a few Be stars from recent papers (see, for example, Steele et al. 1999) have been added. Of course it is impossible to be sure that all the collected objects are definitely of classical type, but the fraction of "doubtful" objects cannot be large (as follows from further discussion, see Sect. 6 and Fig. 2). Thus we can say that the compilation represents the largest ever, more or less homogeneous, sample of classical Be stars. Note that for selection, we collected only stars of III, IV and V luminosity class and the sample does not include any supergiants. Emission line stars of the spectral type A and later are also excluded. No limitation has been made on the level of observed polarization $\left(p_{\text {obs }}\right)$ of the sample stars. Thus we have in hand for statistical study:

1. 497 stars with polarization data;

2. 246 stars with values of near IR excess $E(V-L)$;

3. 151 stars with values of IR excess at $12 \mu \mathrm{m}$, and

4. 463 stars with $v \sin i$ values.

All collected data are presented in Appendix I. It is, of course, of direct interest to investigate the distributions of the observed parameters for the entire sample, but in addition, it is also important to compare the data according to different spectral subgroups. The latter is obviously important to astrophysical understanding but such breakdown also allows investigation of the homogeneity of the total assembly. To obtain statistically representative data sets, we separate the entire sample into four subgroups. Subdividing our list yields 174 stars in the interval OB1.5, 170 stars in the interval B2-B2.5, 147 stars in the interval B3-B5.5, and 135 stars in the interval B6-B9.5. This separation was not justified physically a priori, but it reflects an approximately equal range of effective temperature $T_{\mathrm{e}}\left(\Delta T_{\mathrm{e}} \approx 5000 \mathrm{~K}\right)$ for the last three subgroups.

\section{Data reduction}

Because the main aim of the present investigation is a statistical study of different observed parameters and correlations between them, it is important to minimize any uncertainties and all kind of statistical biases. Since a principal characteristic of Be stars is their temporal variability, it is best to use the mean values of investigated parameters for each star. The averaging was done by the weighting procedure according to the standard errors of individual measurements (see, for example, Smart 1958).

For the discussion of the various parameters, it is useful to construct the relative distributions of observed values in the form of histograms. For this reason it is very important to choose the best binwidths for the distributions. As will be shown below, the chosen binwidths of $0.3 \%, 0^{\mathrm{m}} \cdot 3$ 
and $50 \mathrm{~km} \mathrm{~s}^{-1}$ for polarization, colour and rotational velocity respectively are typically larger than the error associated with the individual measurements and even larger than the range of variability associated with any of the stars. Consider now the reduction of each of the recorded parameters.

\subsection{Reduction of $v \sin i$}

At present, most Be star investigations have used the data of $v \sin i$ mainly from Slettebak (1982) (164 classical Be stars). Other old data are also available and these are supplemented by numerous more recent collections. The only problem in compiling $v \sin i$ values from different sources is that they have different scales and accurate calibrations between the compiled data are required to avoid systematic errors. It is well known that there is a difference between the "new" and "old" Slettebak's scales (Slettebak et al. 1975). First of all we compared the $v \sin i$ data of Slettebak (1975) with those determined or compiled by others for the same stars and calculated least-squares fits to the data. Following this calibration process, we transformed individual values of $v \sin i$ from different sources into the common "new" scale of Slettebak et al. (1975). Stars which after transformation provided strong disparities in their values according to the source catalogue were excluded. Average weighted values of $v \sin i$ and associated standard errors were obtained for each star using the weighting procedure already mentioned above (see Smart 1958). For the case of single measurements, we used the value of a standard error indicated in the reference or adopted a value for the error equal to $10 \%$ of the measured value. This procedure allows exclusion of most systematic and incidental errors and helped to obtain a new expanded homogeneous data set. The determined values of $v \sin i$ for each star with the standard error are presented in Appendix I. It can be seen that for more than $95 \%$ program stars, the standard error is less than $\pm 40 \mathrm{~km} \mathrm{~s}^{-1}$.

\subsection{Reduction of $E(V-L)$}

The values of near IR excesses $E(V-L)$ were calculated by a classical approach using the measurements of observed and normal colour indices and interstellar reddening in the respective photometric bands, this being summarized as:

$E(V-L)=(V-L)_{\mathrm{obs}}-(V-L)_{0}-\left(A_{V}-A_{L}\right)$

where $(V-L)_{\text {obs }}$ and $(V-L)_{0}$ are the observed and normal colour indices and $A_{V}, A_{L}$ are the values of interstellar reddening in the respective photometric bands. We assume that no circumstellar dust environment exists around most of the considered stars and $E(V-L)$ is the excess due to the contribution of circumstellar gas. For most stars the values of $A_{V}, A_{L}$ were calculated assuming a normal extinction law in the form $A_{V}=3.1 \times E(B-V)$, $A_{L} \approx 0.06 A_{V}$ and $E(B-V)=(B-V)_{\mathrm{obs}}-(B-V)_{0}$ (for a detailed discussion, see, for example, Dougherty et al. 1994). In principle, this procedure may lead to the over-dereddening but the error in most cases does not exceed 0.2 and is unimportant, taking into account the chosen histogram binwidth for $E(V-L)$ of $0^{\mathrm{m}} \cdot 3$. The values of $(V-L)_{0}$ and $(B-V)_{0}$ were taken from Straižys (1977). It is easy to show that due to the uncertainty $\sigma_{E(B-V)} \approx 0$ m 05 , the uncertainty in $E(V-L)$ is about $0^{\mathrm{m}} \cdot 2$. Of greater importance is the study of errors due to the range of photometric variability for individual objects. Most classical Be stars are photometric variables on different time scales. Hubert \& Floquet (1998) recently investigated photometric variability of a sample of 289 classical Be stars using the Hipparcos photometric data. They found that most of their program stars (about 33\%) exhibited photometric variability $0^{\mathrm{m}} \cdot 1 \leq \Delta H_{\mathrm{p}} \leq 0^{\mathrm{m}}$.3. Other stars usually show a lower level of photometric variability. Similar conclusions were made recently by Moujtahid et al. (1998) who compiled the values of $\sigma_{V}$ for 50 well known classical Be stars. Moreover, for the calculation of $E(V-L)$, optical and IR data were selected to be as close as possible in time.

\subsection{Reduction of $p_{*}$}

Currently, the intrinsic polarization components have been estimated for only about 90 classical Be stars. In order to obtain a $p_{*}$ value for each star, its interstellar component, $p_{\text {is }}$, needs to be subtracted. This is best done using the "field star method" whereby the local growth of $p_{\text {is }}(D)$ with distance, $(D)$, is first obtained from measurements of stars close to the line of sight of the target star. According to the distance of the considered Be star, an estimate for its $p_{\text {is }}$ component is then made from the calibration and vectorially subtracted using the component Stokes parameters. The selected fields ranged from $1^{\circ}$ to $5^{\circ}$ depending on the number of measured stars in the vicinity. A recent advance for the method has been the improvement in accuracy by the application of Hipparcos data (at least for objects with $D<500 \mathrm{pc}$ ), this being used in the reduction scheme for most of the classical Be stars in our list. Fortunately, the average weighted values of observed polarization, $p_{\text {obs }}$, for most objects in our list together with the associated $\sigma_{p_{\text {obs }}}$ were calculated recently by Heiles (2000), so considerably reducing the amount of laborious work required for the exercise. For a few stars which were investigated polarimetrically more intensively, we estimated $\sigma_{p_{\text {obs }}}$, taking into account the data from recent papers of Quirrenbach et al. (1997), McDavid (1999, 2000) and Ghosh et al. (1999).

Two typical examples of the reduction scheme are presented in Fig. 1. Note that, in some cases, $p(D)$ may not display monotonic growth due to the line of sight passing through interstellar dust clouds with complex structure and different orientation of dust grains. For the cases where the distance to an object could not be determined from the Hipparcos data or the parallax errors were too large, we estimated the distance to a star either from reddening or from an absolute magnitude-spectral 

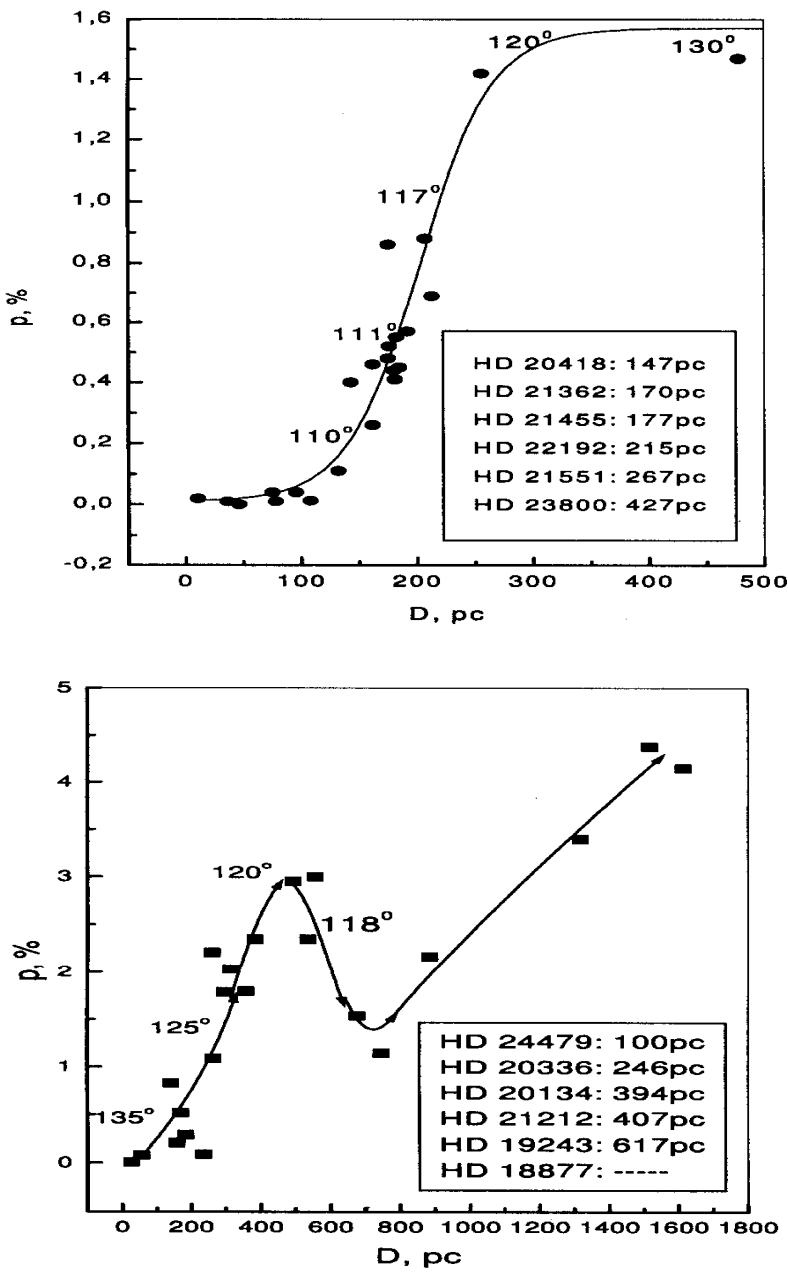

Fig. 1. Dependence between the interstellar polarization and the distance in the $5^{\circ}$ field surrounding a few program stars. The numerical values attached to the curves refer to the position angle of interstellar polarization according to distance

type/luminosity calibration. In a few cases an interstellar component has been estimated by the investigation of the uniformity of polarization vectors in the vicinity of the star. The parameters of $p_{\mathrm{obs}}, p_{\text {is }}$ and $p_{*}$ for the program stars are presented in Appendix II. It is seen that except for a few stars, most values of $\sigma_{p_{\text {obs }}}<0.3 \%$ and thus less than the chosen histogram binwidth.

An independent by-product of this study is the new set of estimates of interstellar polarization components, this being useful for other future investigations.

\section{4. $E(V-L)$, polarization and $v \sin i$ as a function of spectral type}

\subsection{The excess $E(V-L)$ against spectral type}

In Fig. 2a the values of the excess $E(V-L)$ are plotted against spectral type for the entire sample. The following features are immediately obvious. Firstly, there is a clear upper limit for a given spectral type with the maximum value of about 1.8 for B0-B2 stars and with the decrease of near IR excesses toward later than B2 spectral types.
A similar result was obtained by Dougherty et al. (1991) who found that most of late type stars show little or no near IR excess colour but for a significantly smaller statistical sample. However, in contrast to previous studies, we conclude that the decrease of the upper limit of $E(V-L)$ towards late spectral types is well fitted by a function in the form of an exponential decay rather than the linear decay. For the spectral interval from B1 to B9.5 the best fit is described as follows:

$E(V-L)=0.31+1.67 \times \exp \left(-\frac{X-0.70}{2.94}\right)$

where $X$ is the value of the spectral subtype. This fit is indicated by a solid curve in Fig. 2a with $95 \%$ of program stars of a given spectral types located below the curve. Note that approximately the same curve represents the change of the stars' effective temperatures according to spectral subtype. This behaviour is readily understandable, in general terms, as the near IR excess is dependent on the electron density in the circumstellar environment which is in turn a function of stellar temperature. Secondly, the value of IR excesses shows a larger scatter for B1-B3 spectral types. Thirdly, less than $5 \%$ of the investigated stars are above the limit (2). The discussion on the anomalous objects and the distributions of IR excesses for different spectral subgroups of Be stars will be considered in more detail in Sect. 6.

\subsection{Polarization against spectral type}

In Fig. $2 \mathrm{~b}$ the values of $p_{*}$ for the entire sample are plotted against spectral type. This distribution shows a similar behaviour as for near-IR excesses. Firstly, the values show larger scatter for Be stars of earlier spectral types (B0-B3). Secondly, there is an upper limit of $p_{*}$ for a given spectral type. This is represented by a solid curve in Fig. $2 \mathrm{~b}$ below which $95 \%$ of stars of the given spectral type (in the range B3-B9) are located. The upper limit is well described by a function in the form of an exponential decay:

$p_{*}(\%)=0.46+1.74 \times \exp \left(-\frac{X-1.5}{3.22}\right)$

where $X$ is the number defining the spectral subtype. The explanation of this dependence is the same as in the previous section. Thirdly, on average, Be stars of earlier spectral type exhibit larger values of polarization. Fourthly, the maximum values of intrinsic polarization for B0-B3 stars are approximately the same $\left(p_{*} \approx 2 \%\right)$ and the upper limit in polarization for these stars does not correspond to the curve which represents the change of star's effective temperature according to spectral subtypes. This indicates that the behaviour " $p_{*}$ : Sp class" for early type stars is more complicated and there are several physical parameters dictating the levels of polarization. The distributions of $p_{*}$ for different spectral subgroups of Be stars will be considered later in Sect. 7 . 

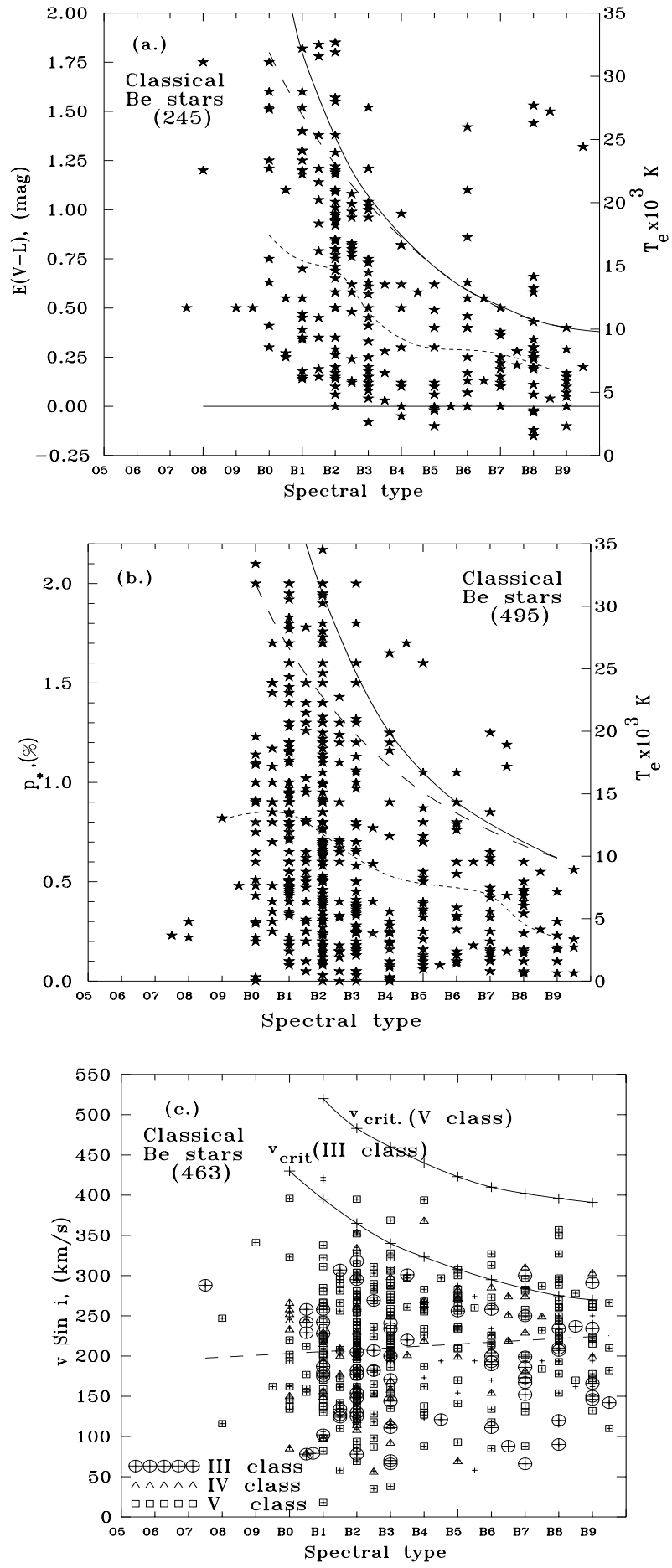

Fig. 2. Near IR excess $E(V-L)$, intrinsic polarization, and $v \sin i$ as a function of spectral type. The upper limit is indicated by the solid curve. The short-dashed curve indicates the average value of the parameters for a given spectral type. The long-dashed curve indicates the value of $T_{\mathrm{e}}$ for a given spectral type

\section{3. $v \sin i$ against spectral type}

In Fig. 2c the values of $v \sin i$ are plotted against spectral type for the entire sample. First impressions suggest that there are no significant differences in $v \sin i$ for the subtypes of Be stars. However, there is a possible trend in the average values in the sense that later spectral type stars rotate faster than early ones. The best linear fit for this trend is:

$v \sin i=(204 \pm 5)+(2.2 \pm 1.2) X$

where $X$ again is the value of the spectral subtype. Note, however, that this trend is not obvious for stars of III luminosity class and it is more pronounced for IV and $\mathrm{V}$ classes:

III: $v \sin i=(190 \pm 14)-(1.1 \pm 2.8) X$
IV: $v \sin i=(183 \pm 14)+(8.0 \pm 3.5) X$
$\mathrm{~V}: v \sin i=(205 \pm 7)+(3.8 \pm 1.6) X$.

The correlations for the above dependencies are small or even absent (correlation coefficients: $r=0.09 \pm 0.06$ for the entire sample, $-0.05 \pm 0.12,0.27 \pm 0.11$ and $0.14 \pm 0.06$ for the III, IV and V classes respectively).

The value of projected rotational velocity shows a large scatter for all Be star subspectral types but with a clear concentration around the values between 200 to $250 \mathrm{~km} \mathrm{~s}^{-1}$. All Be stars exhibit a projected rotational velocities which are less than a critical rotational velocity for the given spectral type. We will investigate the distribution of $v \sin i$ in more detail in the next section.

\section{Distributions of projected rotational velocities}

Two well-known investigations of projected rotational velocities of Be stars were completed 18 years ago (Slettebak 1982 - for a sample of 164 objects; Fukuda 1982 - for a sample of 239 objects). Slettebak noted that there are no strong differences in the rotational characteristics of Be stars of different spectral types or luminosity classes. To the contrary, Fukuda arrived at a different conclusion showing that the middle and later types rotate faster on average than early Be stars, but the influence of luminosity class was not studied. Since then, several attempts to carry out similar kinds of investigation for different samples of classical Be stars have been made with somewhat different conclusions (see, for example, Zorec et al. 1990; Balona 1990; Zorec \& Briot 1997; Grady et al. 1989; Steele 1999 etc.). It is now possible to reassess the situation using the numerous and homogeneous $v \sin i$ data collected and reduced in the present study. We investigate here the distributions of projected rotational velocities of the program stars for four different spectral subgroups (O-B1.5, B2-B2.5, B3-B5.5 and B6-B9.5) and different luminosity classes.

Investigation of a distribution such as $v \sin i$ is a classical problem of mathematical statistics. In the first place, we corrected the constructed histograms from the influence of errors $\left(\sigma_{v} \sin i\right)$ using Eddington's algorithm (see Smart 1958). One of the distributions (viz: for the entire sample of Be stars) is presented in Fig. 3. To compare the distributions, the following parameters should 


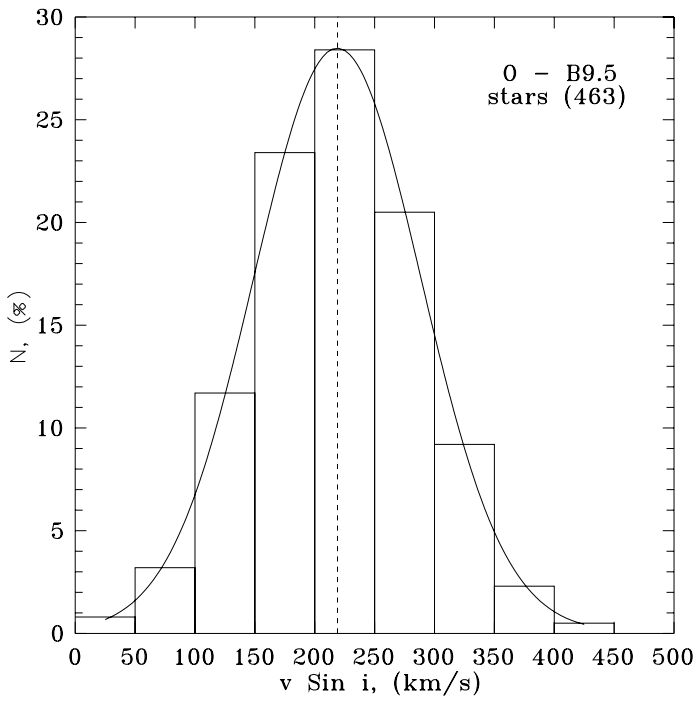

Fig. 3. Observed distribution of $v \sin i$ corrected from errors and its best Gaussian fit for the entire sample of Be stars. The dashed vertical line indicates the mean value of $v \sin i$

be calculated and analyzed: the mean $(\overline{v \sin i})$ and the root mean square deviation $(\sigma)$. To calculate these parameters for a given distribution we used the weighting procedure according to the standard error associated with each contributing measurement (see, for example, Brooks et al. 1994). However, in order to make meaningful comments on the similarity or differences between the distributions, in the first place their distribution functions should be analyzed. As a first step we should try to investigate their "Normality". If the distribution is demonstrably nonNormal, other types of function might then be considered and applied. To test for Normality, we calculated the coefficients of skewness $\left(g_{1}\right)$ and the coefficients of kurtosis $\left(g_{2}\right)$ for each of the distributions. A detailed description of this procedure has been given recently by Brooks et al. (1994) together with the analytical expressions for $g_{1}$ and $g_{2}$. We calculated unbiased estimators of these coefficients with the associated variances (see Table 1) and compared the values with those tabulated in the above mentioned paper for the $95 \%$ and $99 \%$ confidence levels. This analysis indicates immediately that all of the distributions may be considered as Normal at the $99 \%$ confidence level and thus we don't need to invoke other types of distribution functions. At this stage, we cannot say whether the high arithmetical significance of the statistics for Normality carries real physical significance in the sense that it is an expected intrinsic characteristic of the $v \sin i$ distributions of Be stars. Such kind investigations might be the matter of future studies.

The calculated parameters for each of the distributions are given in Table 1. It is of interest also to compare the parameters of distributions of true rotational velocities which are easily calculated by the method suggested by Chandrasekhar \& Münch (1950). In the case of randomly oriented stellar rotation axes, the mean true rota- tional velocity may be calculated by a simple equation: $\bar{v}_{\mathrm{t}}=\frac{4}{\pi} \overline{v \sin i}$.

To test the differences between the dispersions, we can use Fisher's criterion (F-test). Simple calculations indicate that for the III-IV luminosity classes, the differences between the dispersion for the distributions are statistically insignificant at the $95 \%$ confidence level (except late type stars). For the V class, it was found that the difference in $\sigma$ is significant at $95 \%$ confidence level between O-B2.5 and B3-B9 spectral subgroups.

As the next step, we may compare the mean values of $\overline{v_{\mathrm{t}}}$ and the ratio of $\overline{v_{\mathrm{t}}} / v_{\mathrm{c}}$ for selected subgroups. It is well known that the mean true velocities and critical velocities are very different, depending on the luminosity class and spectral class (see for example Zorec et al. 1990). For our purposes we interpolated/extrapolated the values of $v_{\mathrm{c}}$ from Moujtahid et al. (1999) and Zorec (2000) and present them in Table 2 . The values of $\overline{v_{\mathrm{c}}}$ for given spectral subgroups (presented in Table 1) have been calculated taking into account the specific population of stars of different spectral subtypes and luminosity classes in a subgroup. Simple calculations indicate that the differences between the mean values of $\overline{v_{\mathrm{t}}}$ ( or $\overline{v \sin i}$ ) for all spectral subgroups of III-IV luminosity classes are insignificant. There is, however, a pronounced trend in the ratio $\overline{v_{\mathrm{t}}} / v_{\mathrm{c}}$ for these stars. For the $\mathrm{V}$ class, no differences were found between the O-B1.5 and B2-B2.5 subgroups, as well as between subgroups B3-B5.5 and B6-B9.5. Nevertheless, for the subgroups $\mathrm{O}-\mathrm{B} 2.5$ and B3-B9.5, there is strong statistically significant difference at the $99 \%$ confidence level in agreement with the result of Fukuda (1982) who found a clear distinction in $v \sin i$ between B2 and B3 stars. For reference, we present in Table 1 the $\overline{v \sin i}$ data from other sources. The data of Fukuda (1982) (from his Table 5) were transformed into the "new" Slettebak's scale. As follows from Table 1, most early determined values are in agreement (in general) with our data (obtained with 2 to 3 times better statistical quality).

We confirm that late type Be stars rotate faster on average than early types and the trend in the ratio $\overline{v_{\mathrm{t}}} / v_{\mathrm{c}}$ (see Table 1) is also real. We conclude that late type Be stars of all luminosity classes rotate closer to their critical break-up velocity. Whether these differences have affect on the physical characteristics of CS envelopes, or not, will be considered in Sect. 8 .

Finally, it was found that the ratio $\overline{v \sin i} / v_{\mathrm{c}}\left(\right.$ or $\left.\overline{v_{\mathrm{t}}} / v_{\mathrm{c}}\right)$ increases from dwarfs to sub-giants/giants for early spectral type stars (O-B1.5 and B2) though not obvious for middle and late spectral types (see Table 1). This clearly contradicts the results of Steele (1999) who investigated a small sample of Be stars separated into three subgroups according to the luminosity class (III, IV, V-13, 8 and 34 objects respectively). Although our statistical study for sub-giants and giants is also not based on a large sample (135 objects), the behaviour is clearer for the sequence of $\mathrm{V} \rightarrow \mathrm{IV} \rightarrow$ III luminosity classes (see Table 3 ). This phenomenon can be easily explained in terms of a more rapid evolution of high-mass stars and the acceleration of 
Table 1. Mean $\overline{v \sin i}$ values, $(\sigma)$, the coefficients of skewness $\left(g_{1}\right)$ and kurtosis $\left(g_{2}\right)$ of the distributions, true rotational velocities and the ratio of $\overline{v \sin i} / \overline{v_{\mathrm{c}}}$ and $\overline{v_{\mathrm{t}}} / \overline{v_{\mathrm{c}}}$ for different spectral subgroups and different classes of luminosity of Be stars

\begin{tabular}{|c|c|c|c|c|c|}
\hline \multicolumn{6}{|c|}{ III, III-IV classes luminosity } \\
\hline spectral range: & $\mathrm{O}-\mathrm{B} 1.5$ & $\mathrm{~B} 2-\mathrm{B} 2.5$ & B3-B5.5 & B6-B9.5 & O-B9.5 \\
\hline$N$ stars: & 37 & 30 & 28 & 40 & 135 \\
\hline$\overline{v \sin i},\left(\mathrm{~km} \mathrm{~s}^{-1}\right)$ & $203 \pm 11$ & $211 \pm 10$ & $192 \pm 14$ & $207 \pm 10$ & $201 \pm 6$ \\
\hline$\sigma$ & $70 \pm 5$ & $78 \pm 4$ & $89 \pm 6$ & $63 \pm 5$ & $74 \pm 8$ \\
\hline$g_{1}$ & $-0.326 \pm 0.158$ & $0.314 \pm 0.162$ & $0.137 \pm 0.182$ & $-0.234 \pm 0.143$ & $-0.001 \pm 0.042$ \\
\hline$g_{2}$ & $1.998 \pm 0.605$ & $2.441 \pm 0.621$ & $2.454 \pm 0.693$ & $2.653 \pm 0.549$ & $2.412 \pm 0.164$ \\
\hline$\overline{v_{\mathrm{c}}},\left(\mathrm{km} \mathrm{s}^{-1}\right)$ & 456 & 408 & 383 & 320 & 385 \\
\hline$\overline{v \sin i} / \overline{v_{\mathrm{c}}}$ & 0.45 & 0.52 & 0.50 & 0.65 & 0.52 \\
\hline$\overline{v_{\mathrm{t}}},\left(\mathrm{km} \mathrm{s}^{-1}\right)$ & 259 & 269 & 245 & 264 & 259 \\
\hline$\overline{v_{\mathrm{t}}} / \overline{v_{\mathrm{c}}}$ & 0.57 & 0.66 & 0.64 & 0.83 & 0.67 \\
\hline \multicolumn{6}{|c|}{ IV-V, V classes luminosity } \\
\hline spectral range: & $\mathrm{O}-\mathrm{B} 1.5$ & B2-B2.5 & B3-B5.5 & B6-B9.5 & O-B9.5 \\
\hline$N$ stars: & 66 & 78 & 78 & 61 & 283 \\
\hline$\overline{v \sin i},\left(\mathrm{~km} \mathrm{~s}^{-1}\right)$ & $202 \pm 9$ & $207 \pm 8$ & $236 \pm 7$ & $243 \pm 8$ & $229 \pm 4$ \\
\hline$\sigma$ & $80 \pm 9$ & $77 \pm 6$ & $41 \pm 6$ & $58 \pm 5$ & $72 \pm 7$ \\
\hline$g_{1}$ & $0.188 \pm 0.087$ & $0.040 \pm 0.074$ & $-0.586 \pm 0.074$ & $-0.353 \pm 0.094$ & $-0.195 \pm 0.021$ \\
\hline$g_{2}$ & $2.924 \pm 0.339$ & $2.808 \pm 0.290$ & $4.005 \pm 0.290$ & $2.802 \pm 0.365$ & $2.863 \pm 0.083$ \\
\hline$\overline{v_{\mathrm{c}}},\left(\mathrm{km} \mathrm{s}^{1}\right)$ & 527 & 479 & 448 & 398 & 464 \\
\hline$\overline{v \sin i} / \overline{v_{\mathrm{c}}}$ & 0.38 & 0.43 & 0.53 & 0.61 & 0.49 \\
\hline$\overline{v_{\mathrm{t}}},\left(\mathrm{km} \mathrm{s}^{-1}\right)$ & 257 & 264 & 300 & 309 & 292 \\
\hline$\overline{v_{\mathrm{t}}} / \overline{v_{\mathrm{c}}}$ & 0.49 & 0.55 & 0.67 & 0.78 & 0.63 \\
\hline \multicolumn{6}{|c|}{ all classes luminosity (CL) including the stars with undefenite CL } \\
\hline spectral range: & $\mathrm{O}-\mathrm{B} 1.5$ & $\mathrm{~B} 2-\mathrm{B} 2.5$ & B3-B5.5 & B6-B9.5 & O-B9.5 \\
\hline$N$ stars: & 106 & 116 & 121 & 120 & 463 \\
\hline$\overline{v \sin i},\left(\mathrm{~km} \mathrm{~s}^{-1}\right)$ & $201 \pm 7$ & $205 \pm 7$ & $241 \pm 6$ & $225 \pm 6$ & $219 \pm 3$ \\
\hline$\sigma$ & $74 \pm 4$ & $72 \pm 5$ & $59 \pm 5$ & $57 \pm 4$ & $70 \pm 5$ \\
\hline$g_{1}$ & $0.392 \pm 0.055$ & $0.110 \pm 0.050$ & $-0.400 \pm 0.048$ & $-0.234 \pm 0.049$ & $-0.043 \pm 0.013$ \\
\hline$g_{2}$ & $3.333 \pm 0.216$ & $2.666 \pm 0.198$ & $2.995 \pm 0.191$ & $2.971 \pm 0.192$ & $2.877 \pm 0.051$ \\
\hline$\overline{v_{\mathrm{c}}},\left(\mathrm{km} \mathrm{s}^{-1}\right)$ & 502 & 458 & 429 & 362 & 436 \\
\hline$\overline{v \sin i} / \overline{v_{\mathrm{c}}}$ & 0.40 & 0.45 & 0.56 & 0.62 & 0.50 \\
\hline$\overline{v_{\mathrm{t}}},\left(\mathrm{km} \mathrm{s}^{-1}\right)$ & 256 & 261 & 307 & 288 & 280 \\
\hline$\overline{v_{\mathrm{t}}} / \overline{v_{\mathrm{c}}}$ & 0.51 & 0.57 & 0.72 & 0.80 & 0.64 \\
\hline \multicolumn{6}{|c|}{ the data from Slettebak 1982} \\
\hline spectral range: & B0-B1.5 & B2-B2.5 & B3-B5.5 & B6-B9.5 & \\
\hline$N$ stars: & 25 & 35 & 51 & 54 & \\
\hline$\overline{v \sin i},\left(\mathrm{~km} \mathrm{~s}^{-1}\right)$ & 236 & 193 & 221 & 228 & \\
\hline \multicolumn{6}{|c|}{ the transformed data from Fukuda 1982} \\
\hline spectral range: & B0-B1.5 & B2-B2.5 & B3-B5.5 & B6-B9.5 & O-B9.5 \\
\hline$N$ stars: & 69 & 63 & 50 & 57 & 239 \\
\hline$\overline{v \sin i},\left(\mathrm{~km} \mathrm{~s}^{-1}\right)$ & 189 & 196 & 239 & 244 & 214 \\
\hline \multicolumn{6}{|c|}{ the data from Zorec \& Briot 1997} \\
\hline spectral range: & B0-B1.5 & B2-B3 & B4-B7 & B8-B9.5 & \\
\hline$\overline{v_{\mathrm{t}}},\left(\mathrm{km} \mathrm{s}^{-1}\right)$ (II-III, III, III-IV classes) & 230 & 190 & 220 & 130 & \\
\hline$\overline{v_{\mathrm{t}}},\left(\mathrm{km} \mathrm{s}^{-1}\right)(\mathrm{V}$ class $)$ & 240 & 250 & 250 & 280 & \\
\hline \multicolumn{6}{|c|}{ the data from Grady et al. 1989} \\
\hline spectral range: & & & & B6-B9.5 & \\
\hline$N$ stars: & & & & 40 & \\
\hline$\overline{v \sin i},\left(\mathrm{~km} \mathrm{~s}^{-1}\right)$ & & & & 226 & \\
\hline
\end{tabular}


Table 2. The values of $v_{\mathrm{c}}$ for Be stars of different spectral subtypes and class luminosity

\begin{tabular}{lccccccccccccccc}
\hline Spectral type: & O8 & O9 & B0 & B0.5 & B1 & B1.5 & B2 & B2.5 & B3 & B4 & B5 & B6 & B7 & B8 & B9 \\
$v_{\mathrm{c}}$, III class $\left(\mathrm{km} \mathrm{s}^{-1}\right):$ & 500 & 460 & 430 & 412 & 395 & 383 & 365 & 355 & 340 & 325 & 310 & 295 & 284 & 275 & 270 \\
$v_{\mathrm{c}}, \mathrm{IV}$ class $\left(\mathrm{km} \mathrm{s}^{-1}\right):$ & 600 & 560 & 520 & 502 & 483 & 465 & 445 & 435 & 420 & 400 & 386 & 374 & 362 & 352 & 344 \\
$v_{\mathrm{c}}, \mathrm{V}$ class $\left(\mathrm{km} \mathrm{s}^{-1}\right):$ & 626 & 596 & 565 & 540 & 520 & 500 & 483 & 470 & 460 & 442 & 425 & 410 & 400 & 396 & 391 \\
\hline
\end{tabular}

Table 3. The values of $v \sin i / v_{\mathrm{c}}$ for Be stars of different spectral subtypes and classes luminosity

\begin{tabular}{lcccc}
\hline Spectral type: & $\mathrm{O}-\mathrm{B} 1.5$ & $\mathrm{~B} 2-\mathrm{B} 2.5$ & $\mathrm{~B} 3-\mathrm{B} 5$ & $\mathrm{~B} 6-\mathrm{B} 9$ \\
$v \sin i / v_{\mathrm{c}}$, V class: & 0.38 & 0.43 & 0.53 & 0.61 \\
$v \sin i / v_{\mathrm{c}}$, IV class: & 0.43 & 0.49 & 0.50 & 0.64 \\
$v \sin i / v_{\mathrm{c}}$, III class: & 0.46 & 0.56 & 0.51 & 0.65 \\
\hline
\end{tabular}

their rotation. The main sequence lifetime is significantly shorter for B0 stars than for B9 stars (see, for example, Bisnovatyi-Kogan 1989). It may be supposed that most of O-B1.5 and B2 stars of III and IV luminosity classes are possibly in the secondary contraction phase but, on average, the late spectral type stars are not.

\section{Distributions of near and far IR excesses}

As already mentioned, a detailed study of near IR excesses of classical Be stars was undertaken by Dougherty et al. (1994). They used near IR excesses for a sample of 144 Be stars (115 stars with $E(V-L)$ data) for statistical analysis. One of the conclusions was that the excess increases with wavelength and $60 \%$ of Be stars exhibit a significant excess in the $L$ band. They also found a bi-modal distribution of the excesses for the wavelengths greater than $2.2 \mu \mathrm{m}$ but they did not study this behaviour in detail. For this reason, we investigate here the distributions of $E(V-L)$ colour excesses. We have collected the data of $V$ and $L$ photometry for $\approx 200$ stars and, for an additional 40 stars, the values of $E(V-L)$ were estimated by an extrapolation of $J H K$ data. Thus, by essentially doubling the size of the data base over Dougherty et al. (1994), we are able to compare the $E(V-L)$ distributions for different subgroups of Be stars. Note, however, that 6 stars were excluded from the statistical analysis due to their unexpectedly large near IR excess for their spectral type (see Fig. 2a). Some comments on their characteristics and evolutionary status are given below. First of all, the values of near IR excess, $E(V-L)>2^{\mathrm{m}}$, are difficult to explain in a framework of free-free, free-bound emission and such large values require the presence of a dust component in CS environment. Thus, the star HD 181615 (B2Vpe, $\left.E(V-L)=3^{\mathrm{m}} .03\right)$ is likely to contain CS dust. The conclusion on the presence of the dust emission is very likely for the stars HD 50123 (B6IVe, 1.42), HD 51480 (B8e, $\left.1^{\mathrm{m}} .53\right)$, both exhibiting also an excess at $12 \mu \mathrm{m}$ larger than the upper limit for Be stars of a given spectral type (Coté
Table 4. Parameters of distributions of near IR $E(V-L)$ and far IR $E(V-12)$ excesses (in magnitudes) for different subgroups of Be stars

\begin{tabular}{lcccc}
\hline Subgroup: & O-B1 & B2 & B3-B5 & B6-B9 \\
\hline$N$ stars: & 57 & 59 & 58 & 67 \\
\hline$E(V-L)$ & 0.93 & 0.72 & 0.41 & 0.17 \\
\hline$N$ stars: & 29 & 38 & 38 & 40 \\
$E(V-12)$ & 1.90 & 1.59 & 1.39 & 0.72 \\
\hline
\end{tabular}

Parameters of bi-modal Gaussian fits for $E(V-L)$

$\begin{array}{lllll}\text { Peak 1: } \bar{x}_{1} & 0.32 \pm 0.05 & 0.24 \pm 0.03 & 0.16 \pm 0.02 & 0.16 \pm 0.01\end{array}$

$\begin{array}{lllll}\text { Peak 1: } \sigma_{1} & 0.12 \pm 0.02 & 0.14 \pm 0.03 & 0.20 \pm 0.01 & 0.16 \pm 0.02\end{array}$

$\begin{array}{lllll}\text { Peak 2: } \bar{x}_{2} & 1.30 \pm 0.07 & 0.92 \pm 0.04 & 0.80 \pm 0.05\end{array}$

$\begin{array}{llll}\text { Peak 2: } \sigma_{2} & 0.37 \pm 0.08 & 0.37 \pm 0.05 & 0.11 \pm 0.04\end{array}$

Parameters of bi-modal Gaussian fits for $E(V-12)$

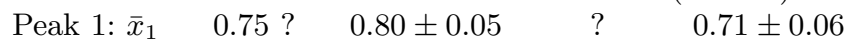

$\begin{array}{lll}\text { Peak 1: } \sigma_{1} & 0.27 \pm 0.04 & 0.30 \pm 0.04\end{array}$

$\begin{array}{llll}\text { Peak 2: } \bar{x}_{2} & 2.07 \pm 0.06 & 2.05 \pm 0.04 & 1.83 \pm 0.05\end{array}$

$\begin{array}{llll}\text { Peak 2: } \sigma_{2} & 0.27 \pm 0.05 & 0.32 \pm 0.04 & 0.30 \pm 0.07\end{array}$

\& Waters 1987). Note that 51 Oph (B9.5V), HD 164906 (B1IV:) and HD 38087 (B5Ve), exhibit unusual far IR excess $\left(3^{\mathrm{m}} \cdot 7,4^{\mathrm{m}} .98\right.$ and $3^{\mathrm{m}} .79$ at $12 \mu \mathrm{m}$ respectively). Relative to classical Be stars, this also indicates the presence of dust emission. An inspection of the lists of Coté \& Waters (1987) and Oudmaijer et al. (1992) shows that only a few Be stars from about 150 objects show excess emission with $E(V-12)>3^{\mathrm{m}}$ and some of them are definitely young objects. Thus, the value $E(V-12) \approx 3^{\mathrm{m}}$ is a limit for free-free and free-bound emission in the CS environment of classical Be stars. The evolutionary status of two other objects: HD 177291 (B8Ve, 1.44) and HD 81357 (B8Ve, $\left.1^{\mathrm{m}} .5\right)$ is not clear. However, the latter star has been classified recently as a binary with an $\mathrm{F} / \mathrm{G}$ secondary (Halbedel 1996) whose light contribution may give an increase to the observed $E(V-L)$ excess.

The distribution of $E(V-L)$ for the entire sample shows the same bi-modal shape behaviour as described by Dougherty et al. (1994) but in a more pronounced form. We performed the same procedure for error correction of the histograms as in the previous section.

Firstly, late spectral type stars exhibit smaller values of $E(V-L)$ on average (see Table 4$)$, this already mentioned in Sect. 4 and noted earlier by Dougherty et al. (1991, 1994), possibly due to a lower electron density in their CS environment. It is obvious from Fig. 4 and in 

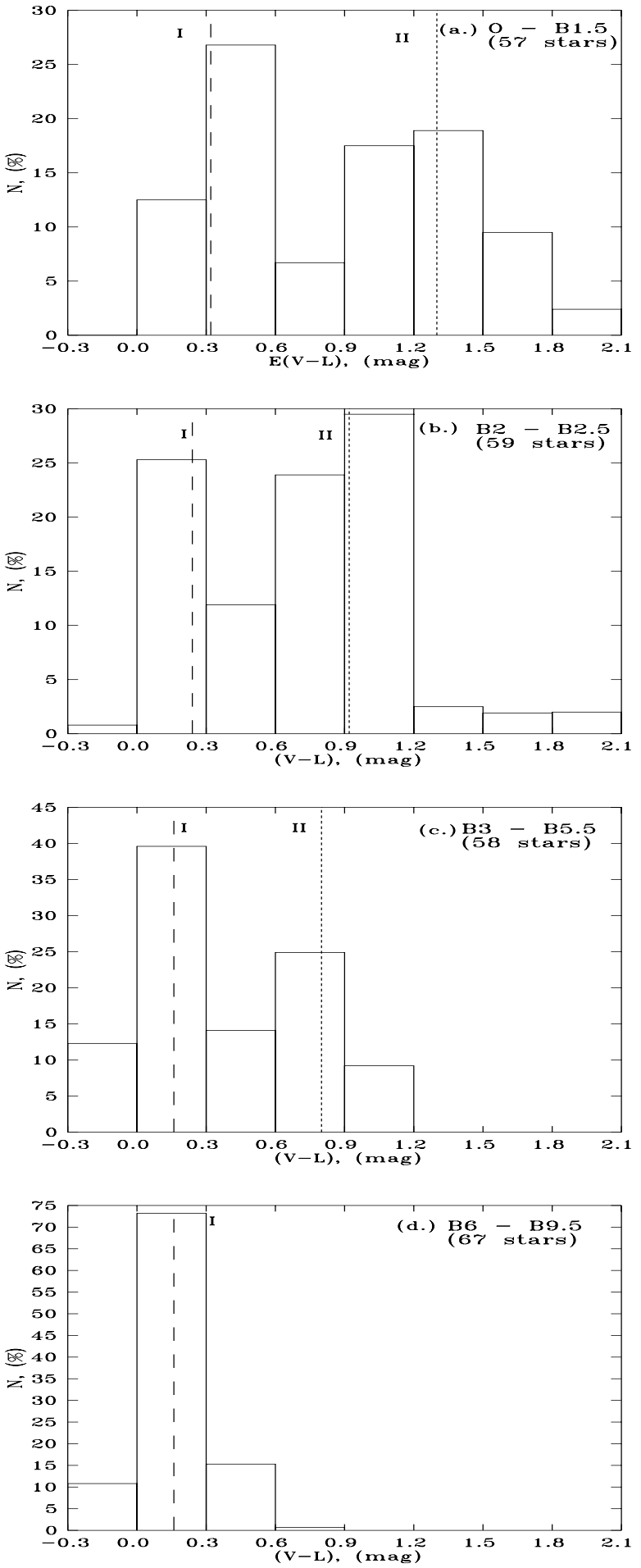

Fig. 4. Observed distributions of $E(V-L)$ (corrected for errors) for different subgroups of Be stars. The short-dashed and long-dashed vertical lines indicate the positions of two peaks for a bi-modal Gaussian distribution

Table 4, that a bi-modal form is present for the first three spectral subgroups of Be stars (O-B1.5, B2-B2.5 and B3-B5.5). For these subgroups, the mean values of $E(V-L)$ corresponding to the first peak are approximately the same, within the length of the sampling step, with $\overline{E(V-L)}_{\text {peak } 1} \approx 0^{\mathrm{m}} \cdot 22 \pm 0^{\mathrm{m}}$.08. Statistical analyses,

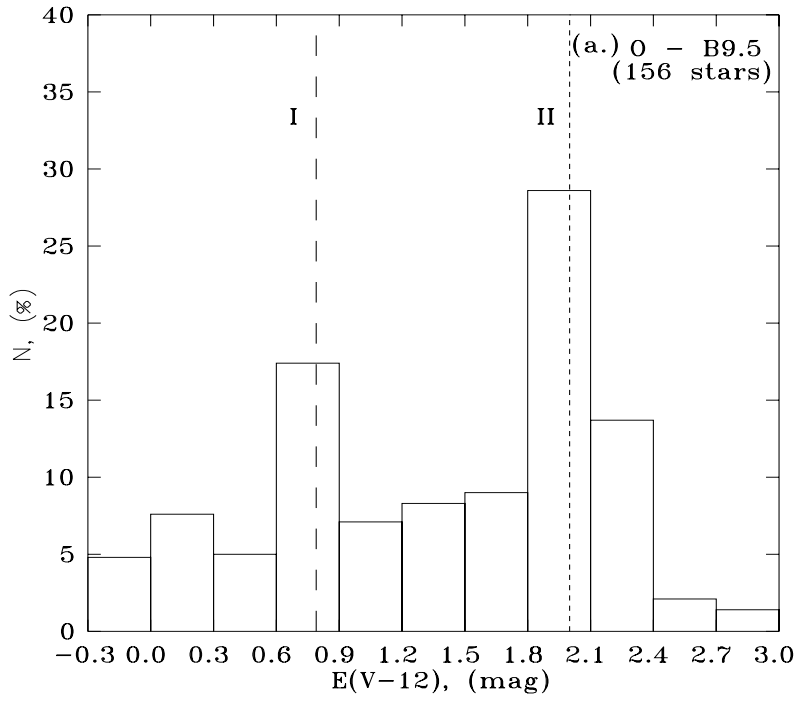

Fig. 5. Observed distributions of $E(V-12)$ (corrected from errors) for the entire sample of Be stars. The short-dashed and long-dashed lines indicate the position of two peaks for a bi-modal Gaussian distribution

similar to those made in previous section, indicate that all the distributions may be well approximated by a bi-modal Gaussian form. This bi-modality for O-B1.5, B2-B2.5 and B3-B5.5 spectral subgroups is confirmed to a $99 \%$ level of confidence. We performed a more detailed study of the B3-B5.5 spectral subgroup and found that a bi-modal distribution is present for B3 type stars with two peaks centered at $0^{\mathrm{m}} \cdot 15 \pm 0^{\mathrm{m}} \cdot 16$ and $0 \cdot 87 \pm 0^{\mathrm{m}} \cdot 21$ ), but bi-modality is not present for B4-B5 stars. For B6-B9.5 stars, the distribution also does not exhibit bi-modality and the mean value of $E(V-L)$ for the best simple Gaussian fit is the same as the first peaks for other subgroups.

The important difference between the various bi-modal distributions is that the second peak has its maximum at different values of $E(V-L)$. The behaviour is that for early spectral types, the second peak appears at larger values of $E(V-L)$. This difference is clearly obvious in a numerical form in Table 4 where the parameters of these distributions are given. Using a t-test and $\mathrm{T}$-test, it is easy to demonstrate that the differences between the positions of the second peaks are statistically significant at the $99 \%$ confidence level. Note that, for the optically thin case, the excess emission in the near IR is proportional to the emission measure which is a function of the mean electron density in formation regions and the ionizing stellar fluxes. It is easy to show from our data that the changes in $E(V-L)_{\text {peak2 }}$ may be well described by a linear function of $\log \overline{T_{\text {eff }}}$ in the form: $E(V-L)_{\text {peak2 }} \approx-11.1+2.78 \log \overline{T_{\text {eff }}}$.

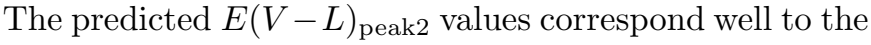
observed values.

It is of interest to compare the behavior of $E(V-$ $L)$ with that for far IR excesses at $12 \mu \mathrm{m}$. Dougherty et al. (1994), with the reference to a private communication of Waters, mentioned the presence of bi-modality of the $E(V-12)$ distribution for Be stars. To check this 

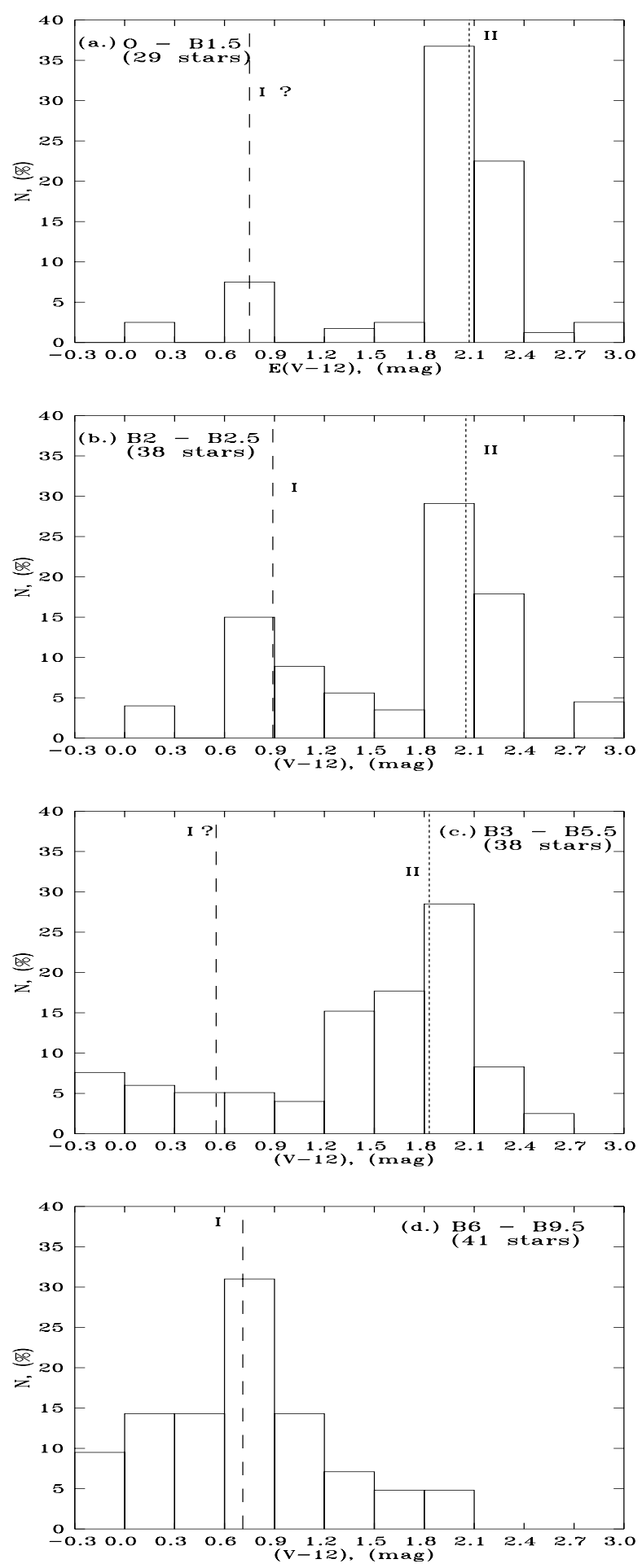

Fig. 6. Observed distributions of $E(V-12)$ (corrected from errors) for different subgroups of Be stars. The short-dashed and long-dashed lines indicate the positions of the two peaks for a bi-modal Gaussian distribution

suggestion we constructed the similar diagrams for $E(V-$ 12). Although bi-modality is clearly seen for the entire sample of Be stars (Fig. 5), it reflects rather the different contributions of early and late type stars. Our sample is insufficiently large to provide any conclusion on bimodality for different spectral subgroups. Slight evidence

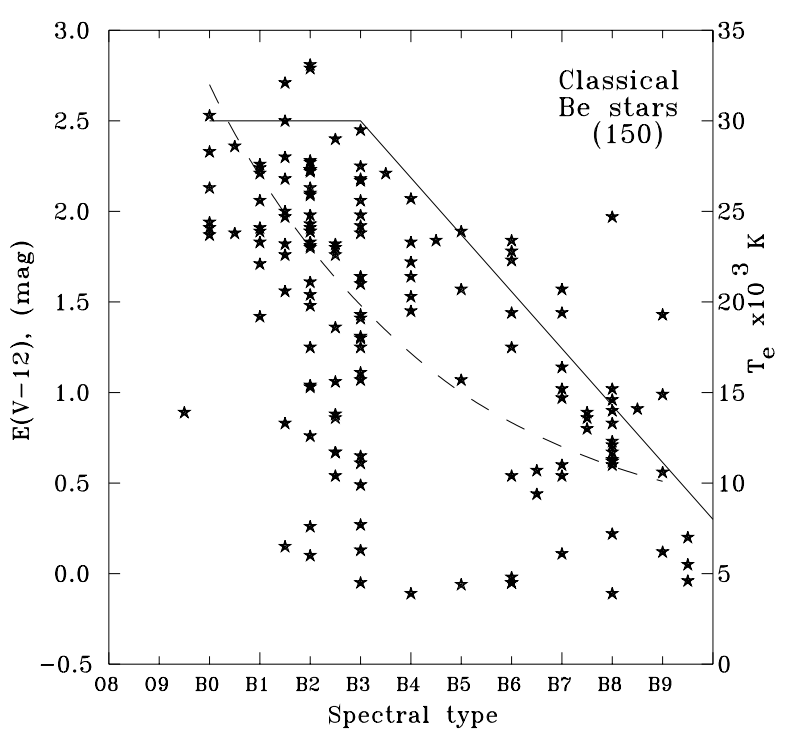

Fig. 7. $E(V-12)$ colour excess versus spectral type for the entire sample of Be stars. The upper limit is indicated by the solid line. The long-dashed line indicates the value of $T_{\mathrm{e}}$ for a given spectral type

of two peaks in the $E(V-12)$ distribution exists only for B2 stars (see Fig. 6). An important point, however, is that the average value of $\overline{E(V-12)}$ and the position of the maximum peak in the distributions is not significantly different between the first three subgroups (see Table 4). Coté \& Waters (1987) considered the far IR data for a sample of 101 Be stars and found the constant upper limit in $E(V-12)$ for stars earlier than B3. The same conclusion follows from the analysis of our data for $150 \mathrm{ob}-$ jects (see Fig. 7). This indicates that the characteristics of the outer parts of CS envelopes are similar for early and middle spectral type stars. For the majority of Be stars, with the excess emission increasing with wavelength, the CS envelopes must become optically thick from at least $\lambda>10 \mu \mathrm{m}$ (Gehrz et al. 1974). With this assumption, for any kind of geometry for the CS environment, the excess $E(V-12)$ is proportional to the effective surface area of the emitting region. It is easy to show that the relation between the upper limit of the excess $E(V-12)$ and $T_{\mathrm{e}}$ will be fairly linear for B3-B9 stars in contrast to the $E(V-L): T_{\mathrm{e}}$ relation. But the behaviour in early type stars is more complicated.

An unresolved point is the nature of the peak 1 in the $E(V-L)$ distributions for early and intermediate spectral type stars. There are several possibilities to explain the value of $E(V-L) \approx 0$ for some objects:

a) there are many Be stars surrounded by small CS envelopes indicated by low emission in $\mathrm{H} \alpha$;

b) the photometric data of $V$ and $L$ used for calculation of the $E(V-L)$ excesses were obtained mainly nonsimultaneously;

c) spectral classification of some early type stars can be dubious. 
Table 5. Differences in the level of intrinsic polarization for the stars in the peak 1 and peak 2 of the $E(V-L)$ distributions

\begin{tabular}{|c|c|c|c|c|c|c|}
\hline Subgroup: & & & & & & \\
\hline $\begin{array}{l}N \text { stars: } \\
\overline{p_{*}}, \%\end{array}$ & $\begin{array}{c}\text { peak } 1 \\
E(V-L)<0^{\mathrm{m}} .85 \\
26 \\
0.57\end{array}$ & $\begin{array}{c}\text { peak } 2 \\
E(V-L)>0.85 \\
26 \\
0.85\end{array}$ & $\begin{array}{c}\text { peak } 1 \\
E(V-L)<0^{\mathrm{m}} \cdot 4 \\
16 \\
0.52\end{array}$ & $\begin{array}{c}\text { peak } 2 \\
E(V-L)>0^{\mathrm{m}} \cdot 5 \\
38 \\
0.63\end{array}$ & $\begin{array}{c}\text { peak } 1 \\
E(V-L)<0^{\mathrm{m}} \cdot 4 \\
26 \\
0.42\end{array}$ & $\begin{array}{c}\text { peak } 2 \\
E(V-L)>00^{\mathrm{m}} .5 \\
20 \\
0.52\end{array}$ \\
\hline
\end{tabular}

For case (a), we would expect a lower $p_{*}$ values for the stars which are concentrated under peak 1. Although the analysis of our data shows some decrease in the polarization level for such stars in comparison with peak 2 , the difference in $\overline{p_{*}}$ for the two peaks is small and insignificant (see Table 5).

For the cases (b) and (c), we would expect a more single-peaked distribution with more extended tails rather than a bi-modal distribution. Finally, it should be remembered that each Be star can show at any time one of the phases - normal $\mathrm{B} \Leftrightarrow \mathrm{Be} \Leftrightarrow$ Be-shell. These phases are characterized by different spectral behaviours (a normal absorption-line spectrum or emission/shell spectrum) due to changes of electron density and/or of geometry of the CS envelopes (Doazan et al. 1988). There is no doubt that during the normal $\mathrm{B}$ phase, the electron density is too small to produce any considerable IR excess. According to Dougherty et al. (1994), for the IR excess in the $L$ band to be detectable, the density of the CS plasma has to be $\approx 10^{-11.6} \mathrm{~g} \mathrm{~cm}^{-3}$. To account for the maximum level of $p_{*} \approx 0.5$ to $1 \%$ observed for the stars under peak 1 (with the above value of density and typical sizes of CS environment $\approx 10$ to $15 R_{*}$ ), in the framework of the disk model, the half-opening angle of the disk cannot be smaller than $10^{\circ}$ to $15^{\circ}$. The important point is that in order to explain a bi-modal character of the distributions, one can suggest that the transition time between the normal $\mathrm{B}$ and $\mathrm{Be} / \mathrm{Be}$-shell phases is much shorter than the duration of the phases themselves. In this case, we can observe an object either at the normal B phase (with $E(V-L) \approx 0$ ) or at the Be/Be-shell phase with $E(V-L)$ values from the distribution under peak 2. In principle, our suggestion is supported by the data of Doazan et al. (1986), who found significant spectral variations in $\theta \mathrm{CrB}$ on a time scale of 3 months when the star changed its phase from shell to normal. At the same time, the duration of the phases in Be stars (or an envelope lifetime) is typically of the order of decades (Cramer et al. 1995; Fox 1991). Our suggestion is consistent also with the hypothesis that the formation of CS envelopes in Be stars is due to short lasting huge mass ejections (Hubert \& Floquet 1998; Moujtahid et al. 1999; Zorec et al. 2000a, 2000b). All the above may be also valid to explain bi-modality in $E(V-12)$ distributions (if it exists). Hubert-Delplace et al. (1981) and Mennickent et al. (1994) noted that the time scale of phase transitions is shorter in early-type Be stars than in late spectral types. Thus we would expect less pronounced bi-modality in late types too, this not being obvious in our data. The apparent absence of bi-modality for late spectral types (B4-B5 and
B6-B9.5) can be explained by the notion that the second peak would be placed toward smaller values of $E(V-L)$. Its possible location is perhaps masked by a combination of the chosen binwidth in association with the small statistical sample.

\section{Distributions of intrinsic polarization for different subgroups of Be stars}

As was noted before, (see Sect. 4.1 and Fig. 2b), Be stars of earlier spectral type tend to exhibit larger values of intrinsic polarization. Thanks to the large bank of calculated values of $p_{*}(\approx 500$ objects $)$, it is possible to investigate the above mentioned behaviour in detail.

Analysis of the intrinsic polarization distribution for classical Be stars was first performed by McLean \& Brown (1978) for a sample of 67 objects. They noted a rapid rise of the distribution toward smaller values of $p_{*}$. The distribution established here (corrected for errors) for a sample of 495 objects (see Fig. 8) is similar to that obtained by McLean \& Brown (1978). However, with the chosen binwidth $(0.3 \%)$, our distribution peaks at $p_{*} \approx 0.4 \%$ with the average weighted value $\overline{p_{*}}=0.68 \%$ and $\sigma_{\mathrm{p}}=0.48 \%$. Our large sample allows us also to study for the first time the distributions of $p_{*}$ for the representative samples of Be stars of different spectral subgroups. These distributions, also error corrected, are shown in Figs. 9a-c. For O-B1.5 stars we used a slightly larger binwidth (0.35\%) because of the possibly larger uncertainties in determination of interstellar components. It is clearly seen that the histograms are significantly different for each spectral subgroup. In contrast to late type stars, the distributions for early spectral types (O-B1.5 and B2-B2.5) do not peak at the value $p_{*} \approx 0 \%$. This result is significant in spite of the relatively large standard deviations (see Table 6). Similar to the analysis for $E(V-L)$ distributions, we performed a more detailed study of the B3-B5 spectral subgroup and found again that such behaviour is present with just B3 stars, but it disappears for B4-B5 stars. A clear difference in the values of $\overline{p_{*}}$ for different spectral subtypes and subgroups is apparent in the sense that there is a decrease in mean values of intrinsic polarization toward late spectral types (see Table 6). Physically this fact has been discussed previously by McLean \& Brown (1978) in terms of less dense and/or less oblate envelopes for later stars. Application of various tests indicates that differences in the mean values of intrinsic polarization for different subgroups of Be stars are statistically significant. McLean \& Brown (1978) found that there are no objects among 
Table 6. Intrinsic and true polarization for different spectral subtypes and subgroups of Be stars

\begin{tabular}{|c|c|c|c|c|c|c|c|c|c|c|c|}
\hline $\begin{array}{l}\text { Spectral type: } \\
\text { No. of stars: }\end{array}$ & $\begin{array}{c}\mathrm{O}-\mathrm{B} 0 \\
47\end{array}$ & $\begin{array}{c}\text { B1 } \\
118\end{array}$ & $\begin{array}{l}\text { B2 } \\
146\end{array}$ & $\begin{array}{l}\text { B3 } \\
58\end{array}$ & $\begin{array}{l}\text { B4 } \\
22\end{array}$ & $\begin{array}{l}\text { B5 } \\
25\end{array}$ & $\begin{array}{l}\text { B6 } \\
20\end{array}$ & $\begin{array}{l}\text { B7 } \\
23\end{array}$ & $\begin{array}{l}\text { B8 } \\
23\end{array}$ & $\begin{array}{l}\text { B9 } \\
11\end{array}$ & \\
\hline $\begin{array}{l}\bar{p}_{*},(\%) \\
\bar{p}_{\mathrm{t}},(\%)\end{array}$ & $\begin{array}{l}0.83 \\
1.25\end{array}$ & $\begin{array}{l}0.85 \\
1.28\end{array}$ & $\begin{array}{l}0.75 \\
1.13\end{array}$ & $\begin{array}{l}0.59 \\
0.88\end{array}$ & $\begin{array}{l}0.52 \\
0.78\end{array}$ & $\begin{array}{l}0.48 \\
0.72\end{array}$ & $\begin{array}{l}0.47 \\
0.70\end{array}$ & $\begin{array}{l}0.45 \\
0.67\end{array}$ & $\begin{array}{l}0.29 \\
0.43\end{array}$ & $\begin{array}{l}0.22 \\
0.33\end{array}$ & \\
\hline $\begin{array}{l}\text { Spectral range } \\
\text { No. of stars: } \\
\bar{p}_{*},(\%) ; \sigma_{\mathrm{p}} \\
\bar{p}_{\mathrm{t}},(\%)\end{array}$ & $\begin{array}{c}\mathrm{O}-\mathrm{B} \\
16 \\
0.84 \\
1.2\end{array}$ & $\begin{array}{l}1.5 \\
0.48 \\
7\end{array}$ & $\begin{array}{c}\mathrm{B} 2-\mathrm{B} 2.5 \\
146 \\
0.75 \quad 0.48 \\
1.13\end{array}$ & & $\begin{array}{r}\text { B3-B5 } \\
105 \\
.55 \quad 0 . \\
0.82\end{array}$ & & & $\begin{array}{r}\mathrm{B} 6 \\
0.37 \\
0\end{array}$ & $\begin{array}{l}39.5 \\
7 \\
0.28 \\
6\end{array}$ & & $\begin{array}{c}\text { O-B9.5 } \\
495 \\
0.68 \quad 0.48 \\
1.02\end{array}$ \\
\hline
\end{tabular}

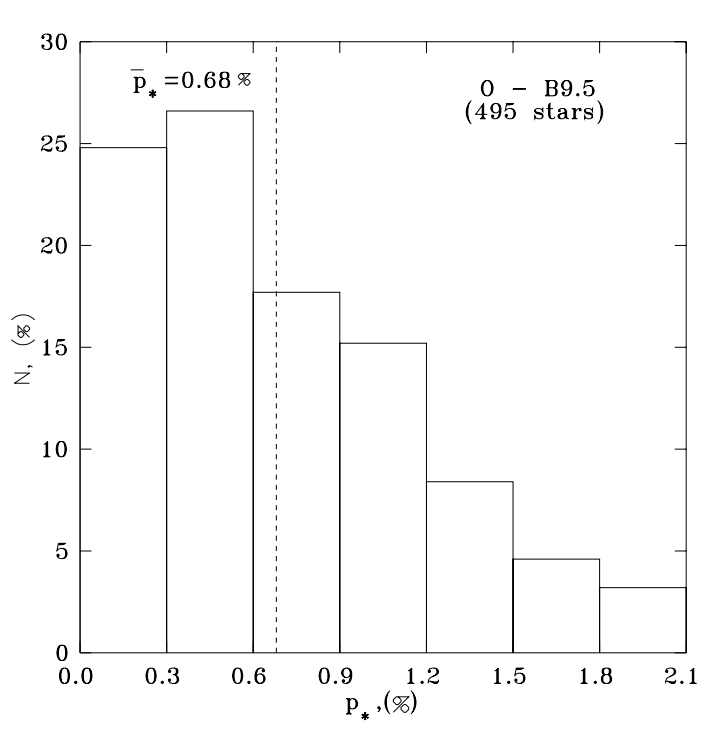

Fig. 8. Distribution of intrinsic polarization (corrected for errors) for the entire sample of Be stars. The vertical dashed line indicates the mean value of polarization

Be stars with the intrinsic polarization in excess $2 \%$. Our data allow us to study this suggestion statistically. Only 5 stars out of 497 objects (i.e. about $1 \%$ ) show $p_{*}>2 \%$ and 5 stars additionally exhibit $p_{*}=2 \%$ (all of them are early type stars B0-B2). Moreover, we conclude that about $95 \%$ of Be stars exhibit intrinsic polarization on the level $0 \%<p_{*}<1.5 \%$. This result is very important because Waters \& Marlborough (1992) noted that in a framework of the single-scattering approximation (and geometrically thin disks, $\Theta \approx 15^{\circ}$ ) it is difficult to obtain high levels of polarization above $2 \%$. The observed level of polarization can be explained either by a disk of large optical depth and a small opening angle or by a wide disk (or an ellipsoidal envelope) of small optical depth. To explain the polarization up to $3 \%$, multiple scattering is considered in the series of the papers of Wood et al. (1996a, 1996b, 1997) and early by Daniel (1980). Nevertheless, there is much evidence that CS envelopes are optically thin, at least up to $\lambda \approx 10 \mu \mathrm{m}$ (see, for example, Gehrz et al. 1974); this also follows from multicolour IR polarimetry of some Be stars by Jones (1979) (as the position angle does not change from the visual to $2.2 \mu \mathrm{m}$ ). From comparisons of position angles of CS disks derived from optical inter-
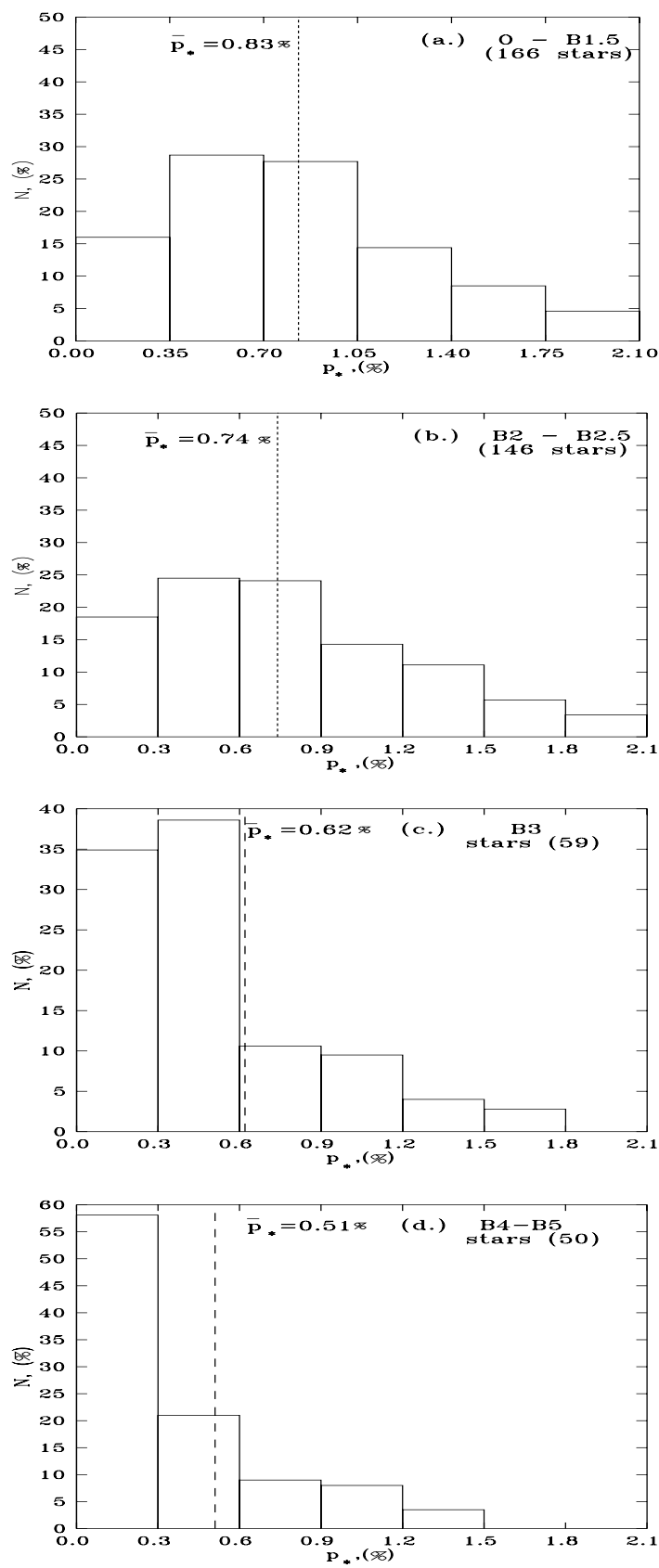

Fig. 9. Distributions of intrinsic polarization (corrected for errors) for different spectral subgroups of Be stars. The vertical dashed line indicates the mean value of polarization 
ferometry and intrinsic position angles of polarization for seven Be stars, Quirrenbach et al. (1997) reached the same conclusion. As follows from our analysis, the percentage of Be stars with $p_{*}>1.5 \%$ is negligible and multiple scattering (and strongly flattened disks: $\Theta<5^{\circ}$ and $\tau>1$ ) may occur only in a few objects. Note that the upper limit in intrinsic polarization can be larger if we consider the true polarization by removing the aspect angle effect, using the method suggested by McLean \& Brown (1978): $\bar{p}_{\mathrm{t}}=\frac{3}{2} \overline{p_{*}}$. For reference, the parameters of true polarization for $\mathrm{Be}$ stars of different spectral subtypes and subgroups are also given in Table 6. Even in this case, however, the fraction of Be stars with $p_{\mathrm{t}}>2 \%$ does not exceed $10 \%$.

The next point to consider is the possible differences in polarization of Be and Be-shell stars. This comparison is quite important as there is active debate on the change in the structure of CS envelopes of Be and Be-shell stars (Marborough et al. 1993; Hanuschik 1996; van Kerkwijk et al. 1995). It was found earlier that for some objects, the level of polarization increases when a star goes through a shell phase (Arsenijevic et al. 1987). Using a small statistical sample, Fox (1993b) noted a little higher level of polarization of Be-shell stars (12 objects, $0.77 \pm 0.41 \%$ ) in comparison with non-shell stars (21 objects, $0.42 \pm 0.39 \%$ ) (note, however, the magnitudes of the errors). On the other hand, Ghosh et al. (1999) found no distinction in polarization level between 24 Be and 5 Be-shell stars. Using the data of Hanuschik (1996) we are able to identify 46 Be-shell stars which have shown shell characteristics at some point in their observed history and compare their behaviour with others. The average values of the parameters for non-shell stars have been calculated using the data from Tables 4 and 6 for the same specific population of stars of different spectral subtypes as in the shell subgroup. As follows from Table 7, no differences (in polarization and IR excesses) exist between Be non-shell and Be-shell stars. A compilation of polarimetric data for Pleione (see WUPPOL 1999) for different phases (Be and Be-shell) also shows the absence of a significant changes in the polarization level. This suggests that the phase transition $\mathrm{Be} \Leftrightarrow$ Be-shell has no strong affect on the parameters describing the inner parts of the disks. Note, however, that all Be-shell stars show significant variability in photometry and polarimetry and the averaging over a long period is not a correct procedure. For this reason, the above discussion can be considered as only a preliminary suggestion. A statistical analysis of the rotational characteristics of Beshell stars is much more informative as their $v \sin i$ values do not depend on the phase changes. In this context it is interesting to investigate an inclination aspect of $\mathrm{Be}$ and Be-shell stars as discussed earlier by Moujtahid et al. (1999). Although the errors are large, the mean viewing angle (" $i$ ") of Be-shell stars derived from the ratio $\overline{v_{\mathrm{t}}} / \overline{v_{\mathrm{c}}}$ is larger on average than that for other Be stars $\left(52^{\circ} \pm 20^{\circ}\right.$ and $40^{\circ} \pm 19$ respectively). Thus, Be-shell stars are viewed closer to the CS disk plane and none of them are observed at the angle less than $30^{\circ}$. On the other hand, there is clear difference in average values of " $i$ " between Be-shell
Table 7. Differences in the observed characteristics of Be-shell and Be stars. The number of stars $(N)$ used to calculate the average values of the parameters is indicated in parentheses

\begin{tabular}{lccc}
\hline & $p_{*}$ & $E(V-L)$ & $E(V-12)$ \\
\hline Be-shell $(N$ stars $)$ & $0.61 \%(44)$ & $0^{\mathrm{m}} \cdot 61(40)$ & $1^{\mathrm{m}} \cdot 49(31)$ \\
non-shell $(N$ stars $)$ & $0.57 \%(448)$ & $0^{\mathrm{m}} .47(203)$ & $1^{\mathrm{m}} \cdot 34(119)$ \\
\hline
\end{tabular}

stars of different spectral subgroups:

O9-B1.5: $42^{\circ} \pm 12^{\circ} \quad$ B2-B2.5: $41^{\circ} \pm 10^{\circ}$

B3-B5.5: $59^{\circ} \pm 18^{\circ} \quad$ B6-B9.5: $55^{\circ} \pm 17^{\circ}$.

If the transition to the Be-shell phase is due to mass loss preferably close to a CS disk plane, the opening angle of CS disks may be smaller for late type stars.

The last point for consideration is a deviation of the distribution peaks from $p_{*}=0 \%$ for early type stars. Although the problems in detection of the peak in a distribution with very small values of $p$ are discussed by McLean \& Brown (1978), the differences detected here are probably real. The first possibility to explain the behaviour is that most early type stars in our sample are more distant objects. The estimates of interstellar components for them are not so precise, so that the displacement from $0 \%$ can be attributed to inaccuracy of its removal. On the other hand, the behaviour can be explained if the suggestion on the randomly oriented rotation axes of envelopes is not true. This point will be discussed in a separate paper. Finally, it may be due to more complicated geometries associated with the CS environments of early-type stars.

\section{Discussion}

An excellent discussion on the problems of analysis of the polarization distribution of classical Be stars has been presented by McLean \& Brown (1978), and a detailed discussion of near and far IR characteristics was made by Coté \& Waters (1987) and Dougherty et al. (1991, 1994). Such kind of theoretical work is beyond the purpose of our paper and we will discuss some of our results only briefly. Our very large statistical sample allows us to explore possible correlations between the parameters discussed here and to make some suggestions on CS envelope geometries.

First, no clear correlations exist between the intrinsic polarization and near IR excesses neither for a sample of 209 stars (see Fig. 10a) nor for different spectral subgroups. This negative result is very important because it indicates that the regions in CS envelopes which are responsible for generating the polarization and near IR excesses are different. Note that Coté \& Waters (1987) and Waters \& Marlborough (1992) found and discussed a possible correlation between the polarization and far IR excesses at $12 \mu \mathrm{m}$, but on the basis of very small sample (46 stars). To study this suggestion, we investigated the correlation between the values of polarization and the excesses at $12 \mu \mathrm{m}(E(V-12))$ for 125 Be stars. The data of $E(V-12)$ were taken from Coté \& Waters (1987) and 

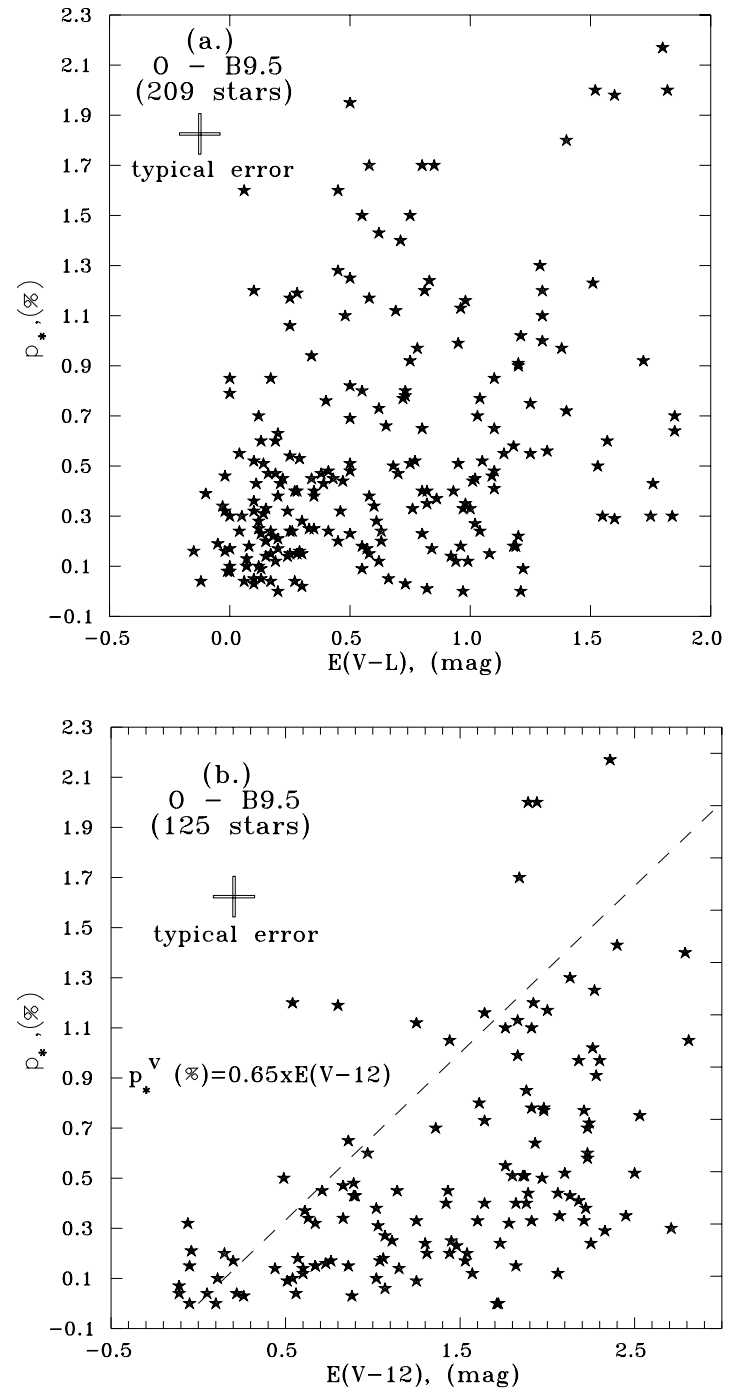

Fig. 10. Correlation between near a) and far b) IR excesses and intrinsic polarization for the entire sample of Be stars

Oudmaijer et al. (1992). A comparison of Figs. 10a and $10 \mathrm{~b}$ indicates that, in contrast to near IR excesses, the relation between the intrinsic polarization and far IR excesses, with a factor three improvement of sample size, really exists in the form of a triangular shaped distribution similar to that found by Coté \& Waters (1987). With a few exceptions (6 stars, three of them Be-shell), there are no stars with high polarization and low far IR excesses. The well defined upper limit is described by the empirical relation:

$p_{*}^{V}=0.65 \times E(V-12)$.

Note that Coté \& Waters (1987) plotted $B$ band values of $p_{*}$ against $E(V-12)$ and the upper limit for their dependence is described by $p_{*}^{B}=0.83 \times E(V-12)$. The ratio of $p^{B} / p^{V}$, however, is virtually constant for all classical Be stars (see, for example, Ghosh et al. 1999) and is equal to $1.28 \pm 0.09$. Taking into account this ratio, we conclude that the upper limits for our dependence and for the dependence of Coté \& Waters (1987) are exactly the same. Reasons for the lack of correlations between $p_{*}$ and

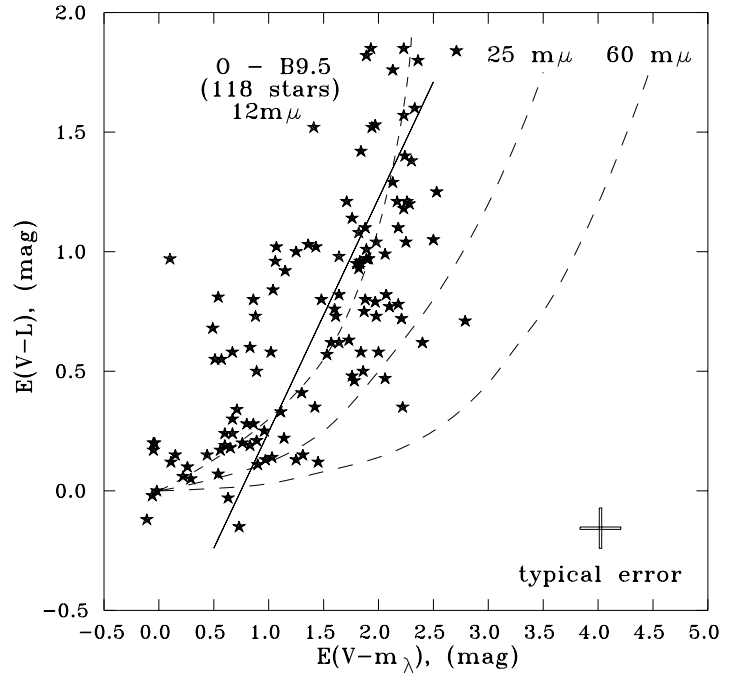

Fig. 11. Correlation between near and far IR excesses for the entire sample of Be stars. The dashed lines represent a possible non-linear fit for the observational data for $E(V-L)$ : $E(V-$ $\left.m_{\lambda}\right)$ dependencies where $m_{\lambda}$ is the magnitude at $12 \mu \mathrm{m}, 25 \mu \mathrm{m}$ and $60 \mu \mathrm{m}$ respectively. The data points marked in this figure concern $E(V-L)$ and $E(V-12)$ data

$E(V-L)$ in contrast to the $p_{*}: E(V-12)$ triangular distribution is not clear. As noted by Poeckert \& Marlborough (1976) and Coté \& Waters (1987), the bulk of the polarization is produced in layers within $<3 R_{*}$, while the $12 \mu \mathrm{m}$ excess can originate from layers that are further out. However, it was shown recently by Fox (1993b), that two parameters are important for continuum linear polarization in Be stars: the envelope oblateness and the location of the inner boundary of the cool extended envelope (which is typically $\approx 5 R_{*}$ ). The behaviour seen in Fig. 10b does not contradict to the suggestion that $p_{*}$ originates in those regions of CS envelopes which are optically thin. It may reflect a link between far IR excesses (or polarization) and the size of CS envelopes.

Note that although the correlation between near and far IR excesses clearly exists (with a correlation coefficient of $r=0.65 \pm 0.06$ ), there is a significant scattering of the data points (see Fig. 11). The large spread is difficult to explain if near and far IR excesses arise in the same gaseous disks and have the same nature (free-free and free-bound radiation). The correlation coefficients for different spectral subgroups show an increase toward late spectral type stars $(r=0.36 \pm 0.14,0.55 \pm 0.14$ and $0.66 \pm 0.11$ for B2, B3-B5 and B6-B9 respectively). For O-B1.5 stars, $r=0.71 \pm 0.14$ but the statistical sample is too small (13 objects). Moreover, a visual inspection of Fig. 11 leads to the suggestion that the dependence $E(V-L): E(V-12)$ is not linear and there is a more rapid increase of $E(V-L)$ in the region of large $E(V-12)$ values. The same conclusion follows from the consideration of the $E(V-L)$ : $E(V-25)$ and $E(V-L): E(V-60)$ dependencies. In contrast, the relations between the excesses at $12 \mu \mathrm{m}, 25 \mu \mathrm{m}$ and $60 \mu \mathrm{m}$ are quite linear with correlation coefficients $r \approx 0.8$ to 0.9 . 
On the one hand, the behaviour can be due to the fact that CS envelopes are optically thin at $3.5 \mu \mathrm{m}$ but optically thick at $\lambda>12 \mu \mathrm{m}$ (Gehrz et al. 1974). This may lead to the behaviour indicated for $E(V-L): E\left(V-m_{\lambda}\right)$ dependencies. The theoretical interpretation of these dependencies is beyond the purpose of our paper note, however, that a similar behaviour was discussed theoretically by Dougherty et al. (1994). The regions of large $E(V-L)$ and $E(V-12)$ values in Fig. 11 are occupied mainly by early type stars (see Fig. 2a and Coté \& Waters 1987). These hotter stars can ionize larger volumes of CS matter, leading to the increase in $E(V-L)$ excesses). Poeckert \& Marborough (1982) noted that the dramatic change in ionization structure of CS envelopes is obvious around $T_{\mathrm{e}} \approx 15000$ to $20000 \mathrm{~K}$ corresponding to spectral types B4-B6. At the same time the effective surface area of the emitting regions which are responsible for the excess $E(V-12)$ for B0-B5 stars does not change strongly (see previous section). On the other hand, it is possible to suggest the presence of an additional component to CS envelopes for early spectral type objects close to the star which may be responsible for an increase of near IR excesses. The absence of this second component in late type stars leads to the increase of correlation coefficients for $E(V-L): E(V-12)$ dependence from B2 to B9 stars.

Another important negative result is the absence of any correlation between near-far IR excesses and $v \sin i$ for 220 and 118 Be stars respectively (see Figs. 12a,b) (and for any spectral subgroup considered separately). This behaviour may result from the small optical depth of CS environment for the majority of Be stars in the near IR. Another possibility to account for this behaviour is a suggestion of either a more spherical geometry for the CS environment where the near IR emission arises or the presence of two independent regions - a disk component and a spherical component or polar lobes, both responsible for the near IR emission. The suggestion on two distinct regions in CS environment in classical Be stars (a diffuse polar stellar wind and a dense equatorial disk) is discussed currently in literature (see, for example, Porter, 1998 and references therein). Moreover, in context of polarization modeling, Fox (1991) supposed that for some Be stars the polar region can be the dominant source of free electrons. To minimize the uncertainties due to mixing in a same diagram involving stars with different masses and luminosity classes, we re-construct Fig. 12 using the ratio $v \sin i / v_{\mathrm{c}}$ instead of the values of $v \sin i$. However, as follows from Fig. 13, no correlations appear even with this adjustment. As was noted above, however, CS envelopes are optically thick at $12 \mu \mathrm{m}$. In the framework of this assumption, strongly flattened disks would produce high colour excesses for the case of pole-on orientation and significantly lower excesses at high inclinations (edgeon). For the geometrically thick disks (with $\Theta>50^{\circ}$ ) we would expect an opposite behaviour, namely high excesses at $i=90^{\circ}$ and lower excesses at $i=0^{\circ}$. The absence of correlations between $E(V-12)$ and $v \sin i$ indicates that the effective surface area of emitting regions is approxi-
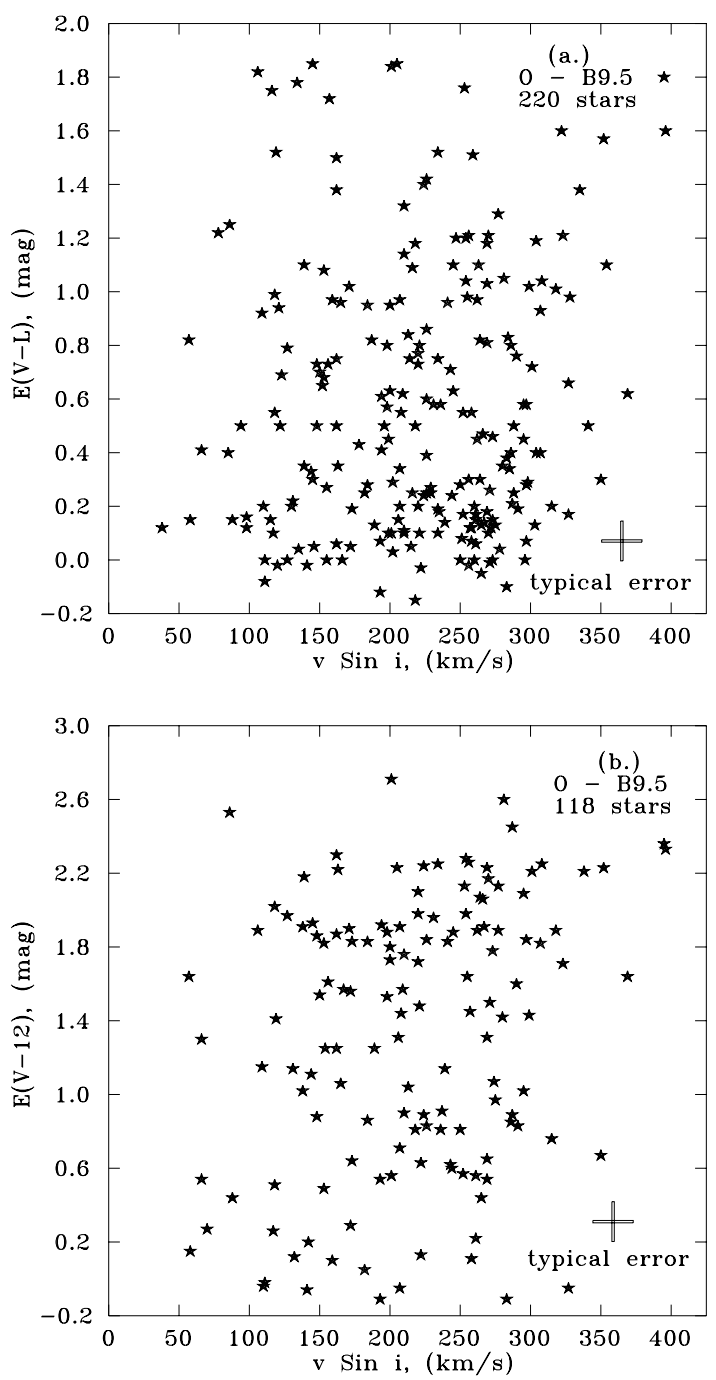

Fig. 12. Correlation between near a) and far b) IR excesses with $v \sin i$ values for the entire sample of Be stars

mately equal for pole-on $\left(S_{1}\left(i=0^{\circ}\right) \simeq \pi R^{2}\right)$ and edge-on $\left(S_{2}\left(i=90^{\circ}\right) \simeq 2 \pi R^{2} \tan \Theta\right)$ cases, where $R$ is the radius of the disk and $\Theta$ is its half-opening angle. Regarding the disk-like structure, from simple geometrical considerations, this is realized for disks with a half-opening angle $\Theta \approx 25^{\circ}$. Such behaviour can never be obtained for ellipsoidal envelopes as the effective surface area (and thus $E(V-12))$ would be always larger for a pole-on orientation! In addition, note that according to Fox $(1991,1994)$ optically thin ellipsoidal envelopes are unable to produce polarizations in excess of $1.2 \%$. Thus, the implication of this configuration of CS envelopes is at least questionable. Assuming $\sigma_{E(V-12)} \approx 0 \mathrm{~m} \cdot 2$, for the difference in $E(V-12)$ not to be detectable at $3 \sigma$ level, the ratio of the effective surface areas for pole-on and edge-on disks cannot exceed 0.6. With this ratio the range of the half-opening angle of CS disks is $10^{\circ}<\Theta<40^{\circ}$. Waters (1986) showed that for a disk with $\Theta=10^{\circ}$, the effect of inclination on the excess flux cannot be negligible for $i>50^{\circ}$. Our estimate of $\Theta$ is in agreement with that of Waters (1986) and Hanuschik (1996) but it clearly contradicts theoretical predictions for 

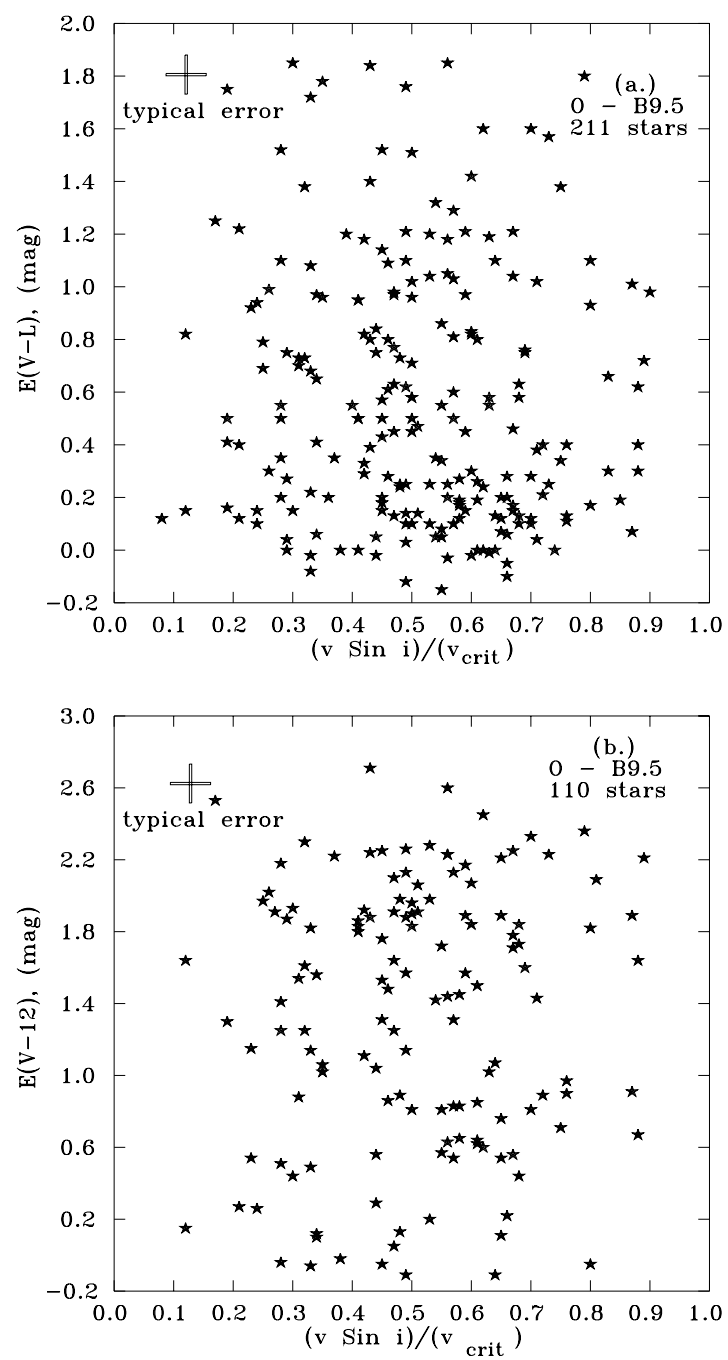

Fig. 13. Correlation between near a) and far b) IR excesses with the ratio of $v \sin i / v_{\mathrm{c}}$ for the entire sample of Be stars

WCD models of Bjorkman \& Cassinelli (1993) and Owocki et al. (1994). De Araújo et al. (1994) have determined the angle of a CS envelope where the density has decreased by a factor of 1 /e with respect to the equatorial value as about 17.5 which is also in agreement with our data. Our large statistical sample allows us to suggest that any uncertainties due to non-simultaneous observations and/or variability of observed parameters should not affect the results significantly and the lack of any strong correlations is a real outcome.

However, if all Be stars are surrounded by CS disks, correlations might be expected between the intrinsic polarization and projected rotational velocities. This dependence was first investigated by McLean \& Brown (1978) and recently for a larger sample of stars by Yudin (2000). In both cases, the relation between $p_{*}$ and $v \sin i$ shows a triangular distribution but with very different boundaries. According to McLean \& Brown (1978), the polarization exhibits a maximum scatter for the largest values of $v \sin i$. In contrast, Yudin (2000) found that $p_{*}$ reaches maximum values for stars with projected rota- tional velocities in the intermediate range of $150 \mathrm{~km} \mathrm{~s}^{-1}$ $<v \sin i<250 \mathrm{kms}^{-1}$ (Feature 1) and there are none (with a few exceptions) with large polarization among the stars with relatively low $v \sin i\left(<100 \mathrm{~km} \mathrm{~s}^{-1}\right)$, (Feature 2) and very high $v \sin i\left(>300 \mathrm{~km} \mathrm{~s}^{-1}\right)$, (Feature 3$)$. Note that Yudin (2000) used the data of intrinsic polarization for about 90 objects and his result is well confirmed in the present study for the largest list of intrinsic polarization of classical Be stars currently available (337 objects, see Fig. 14). The distribution constructed here has a peak at the value $v \sin i \approx 200 \mathrm{~km} \mathrm{~s}^{-1}$ and the boundary lines are described by:

$p_{*}(\%)=0.01(v \sin i) \quad$ for $0<v \sin i<200 \mathrm{kms}^{-1}$ and $p_{*}(\%)=4-0.01(v \sin i)$ for $200<v \sin i<400 \mathrm{kms}^{-1}$.

The behaviour corresponding to Feature 2 has already been discussed in terms of high inclinations of the envelope symmetry axis to the line of sight (McLean \& Brown 1978). While the polarization by scattering in any optically thin axisymmetric CS envelope is proportional to the optical depth and $\sin ^{2} i$, the increased scatter in the $p_{*}$ : $v \sin i$ diagram would be observed toward the larger values of $v \sin i$ (Feature 1). However, polarization of about $2 \%$ is the limit in the canonical model adopted for Be stars which is well confirmed in our study. Fox (1991), however, showed theoretically that the inclination dependence is more complicated than in the model of Brown \& McLean (1977) and that the expression $p_{*} \propto \sin ^{2} i$ no longer holds. Again, to minimize any uncertainties due to mixing of stars with different stellar masses and luminosity classes, we re-construct Fig. 14 using the ratio $v \sin i / v_{\mathrm{c}}$ instead of the values of $v \sin i$. The same triangular shaped distribution is apparent in Fig. 15. The peak of the distribution is at the value $v \sin i / v_{\mathrm{c}}=0.41$, corresponding to the value $v_{\mathrm{t}} / v_{\mathrm{c}} \approx 0.52$. The boundary lines are described as follows: $p_{*}(\%)=4.88\left(v \sin i / v_{\mathrm{c}}\right) \quad$ for $0<\left(v \sin i / v_{\mathrm{c}}\right)<0.4$ and $p_{*}(\%)=3.39-3.39\left(v \sin i / v_{\mathrm{c}}\right)$ for $0.4<\left(v \sin i / v_{\mathrm{c}}\right)<1$.

Finally, note that less than $3 \%$ of Be stars are located outside of the triangular distribution.

Let us consider now a few hypothesis in context to Feature 3. First, to account for this behaviour, it must be remembered that a rapidly rotating Be star (with the value of $v \sin i \approx 300-400 \mathrm{~km} \mathrm{~s}^{-1}$ ) is at the stability limit and has a large coefficient of non-sphericity. It is well known that the rapid rotation of the star produces two effects, namely flattening of the star at the poles and, secondly, a gravitationally induced darkening of the equatorial region. Both of these effects contribute to a polarization whose direction is perpendicular to the polar axis i.e., orthogonal to the vector of polarization arising in an optically thin CS gaseous disk (Bjorkman \& Bjorkman 1994). Consequently, the observed polarization, being the vector sum of the two components (CS polarization and star's photospheric polarization), tends to diminish toward the highest $v \sin i$ values, beginning at some limiting value, this leading to the appearance of the "triangular" distribution of $p_{*}: v \sin i$. Note however, that the depolarizing effect is small, $\left(\Delta p_{*}<0.3 \%\right.$, see 


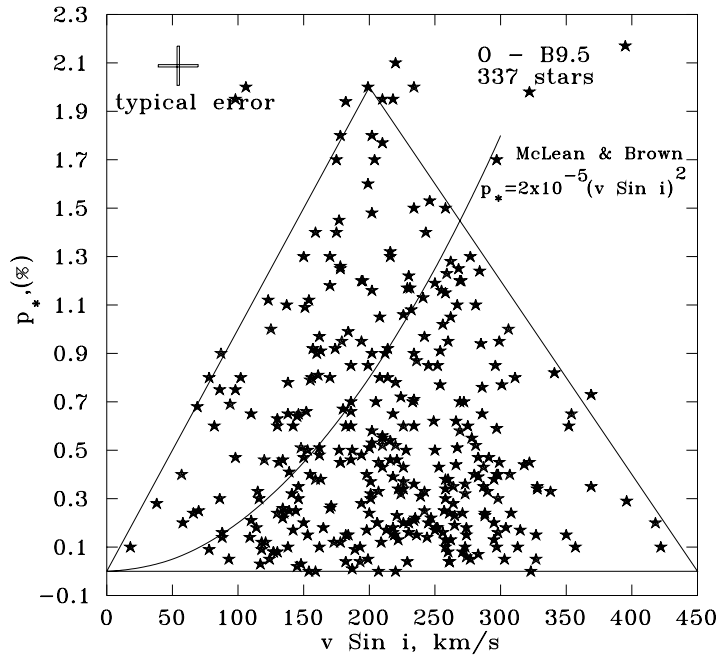

Fig. 14. Correlation between $p_{*}$ and projected rotational velocities for the entire sample of classical Be stars

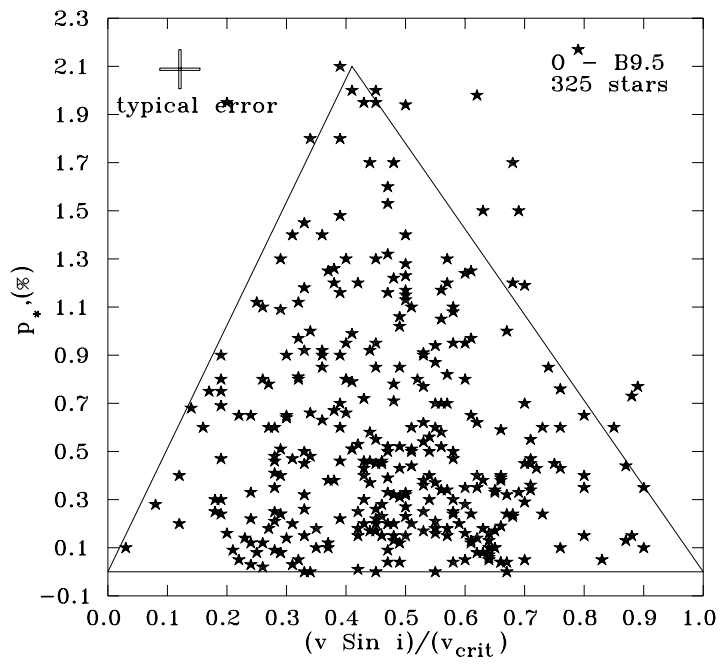

Fig. 15. Correlation between $p_{*}$ and the ratio of $v \sin i / v_{\mathrm{c}}$ for the entire sample of classical Be stars

Bjorkman \& Bjorkman 1994), especially taking into account that the decrease of $p_{*}$ is clearly obvious from the relatively small value $v_{\mathrm{t}} / v_{\mathrm{c}} \approx 0.6$ (see further discussion). Moreover, scattering of stellar polarized radiation in a CS envelope would lead to appearance of circular polarization (also true for multiple scattering) but this is not detected in Be stars at the moment.

The second possibility to account for the above mentioned behaviour is that the triangular shaped distribution might be the result of a combination of two facts established above namely: larger mean projected rotational velocities and smaller absolute values of polarization for late spectral type stars. In this case, most late spectral type stars will be located at the lower right side of the diagram that tending to the decrease of polarization toward larger values of $v \sin i$ for the sample of Be stars. However, a similar diagram constructed with just B6-B9.5 stars again shows the same behaviour with a triangular distribution - as well as for the other spectral subgroups
Table 8. Location of the peaks in $p_{*}: v \sin i / v_{\mathrm{c}}, p_{*}: v_{\mathrm{t}} / v_{\mathrm{c}}$ distributions and the average values of $\overline{v \sin i} / \overline{v_{\mathrm{c}}}, \overline{v_{\mathrm{t}}} / \overline{v_{\mathrm{c}}}$ for different spectral subgroups of Be stars

\begin{tabular}{cccccc}
\hline & O-B1 & B2 & B3-B5 & B6-B9 & O-B9 \\
$\left(v \sin i / \overline{v_{\mathrm{c}}}\right)_{\text {peak }}$ & 0.33 & 0.41 & 0.47 & 0.56 & 0.41 \\
$\overline{v \sin i} / v_{\mathrm{c}}$ & 0.40 & 0.45 & 0.56 & 0.62 & 0.50 \\
$\left(v_{\mathrm{t}} / \overline{v_{\mathrm{c}}}\right)_{\text {peak }}$ & 0.42 & 0.52 & 0.60 & 0.71 & 0.52 \\
$\overline{v_{\mathrm{t}}} / v_{\mathrm{c}}$ & 0.51 & 0.57 & 0.72 & 0.80 & 0.64 \\
\hline
\end{tabular}

of Be stars treated separately. Therefore, the common behaviour is attributed to rapid rotation independently on spectral subtypes.

An alternative suggestion is that the stars with a rapid rotation may form narrower CS gaseous disks. Because the generated polarization is proportional to the ratio of thickness to length of the disk (or to an opening angle, see, for example, Dolginov et al. 1995), such narrower disks produce a significantly lower level of polarization due to increased dilution by unpolarized starlight. Inasmuch as we exclude from consideration multiple scattering, ellipsoidal envelopes, geometrically thick and strongly flattened disks, let us discuss now the optically thin CS disks with $10^{\circ}<\Theta<40^{\circ}$. For disk-like envelopes the maximum degree of polarization $\left(p_{*} \approx 1.5-2 \%\right.$ for the optical depth $\tau \approx 0.5$ ) occurs at $\Theta \approx 35^{\circ}$ (Waters \& Malborough 1992; Fox 1993a, - for single-scattering, plus attenuation) that is similar to the above derived upper limit of $\Theta$. As follows from the theoretical calculations of Fox (1993b, 1994) and Waters \& Malborough (1992), a decrease of $\Theta$ from $35^{\circ}$ to $10^{\circ}$ leads to the decreasing in polarization from $1.5-2 \%$ to $0.5-0.7 \%$. In addition, the above mentioned authors showed that polarization reaches a maximum at the inclination angle $i \sim 85^{\circ}$ rather than at $i \sim 90^{\circ}$. Thus, for exactly edge-on orientation of CS envelopes the observed polarization is reduced by about $0.3-0.5 \%$. Taking into account the star's non-sphericity, a combination of these three effects can reduce the intrinsic polarization to zero for stars with rapid rotation, viewed edge-on. Although the models of disk formation around early-type stars currently discussed in the literature (rotation induced bistability and wind compressed disks) cannot explain the existence of CS disks with $\Theta>5^{\circ}$, in both models the opening angle of a disk decreases with the increase of the ratio $\overline{v_{\mathrm{t}}} / v_{\mathrm{c}}$.

An interesting point is that the peak in the distribution $p_{*}: v \sin i / v_{\mathrm{c}}$ is different for our spectral subgroups (see Table 8). In the framework of the above suggestion, the compression of CS disks which affects the depolarization, sets in for lower values of $\overline{v_{\mathrm{t}}} / v_{\mathrm{c}}$ for early type stars. However, this hypothesis requires a more detailed theoretical study.

Finally, note that our study is essentially just one of statistical analysis only of some of the observational characteristics of Be stars and most physical explanations are only a simple sketch. To construct a self-consistent model of Be phenomena, a detailed theoretical interpretation of 
the new results presented here and earlier results well confirmed in the present study now needs to be undertaken.

\section{Conclusions}

A statistical analysis of some observational characteristics (polarization, projected rotational velocities and near-far IR excesses) for the largest currently available list of classical Be stars (627 objects) has been completed. Our main conclusions may be summarized as follows:

1. Polarization and near IR excesses of Be stars plotted against spectral types show the same behaviour, namely a maximum mean value for B1-B2 spectral types with a decrease toward late spectral types;

2. Projected rotational velocities for the different spectral subgroups of Be stars are statistically significantly different. We confirmed that for luminosity class V, late spectral type stars rotate faster on average and the ratio of $\overline{v \sin i} / v_{\mathrm{c}}$ for them is larger than for early spectral types. For the III-IV luminosity classes, no significant differences exist between $\overline{v \sin i}$ but the ratio of $\overline{v \sin i} / v_{\mathrm{c}}$ is also larger for late spectral type subgroups. All the above confirm well the results of previous studies;

3. Analysis of the data allows us to conclude that for $E(V-L)$ excesses, there is a bi-modal distribution for O-B1.5, B2-B2.5 and B3-B5.5 spectral subgroups. Such a behaviour does not appear for late spectral types (B6-B9.5). The second peak in $E(V-L)$ distributions transits to the lower values from early to late spectral type subgroups;

4. We conclude that about $95 \%$ of Be stars exhibit intrinsic polarization on the level $0 \%<p_{*}<1.5 \%$;

5. No correlation is found between $p_{*}$ and $E(V-L)$. In contrast, a clear relation between $p_{*}$ and far IR excesses at $12 \mu \mathrm{m}$ is confirmed;

6. No correlation is observed between $E(V-L), E(V-12)$ and $\overline{v \sin i}$ or $\overline{v \sin i} / v_{\mathrm{c}}$

7. A clear relation between the intrinsic polarization and $v \sin i$ (as well as for $p_{*}-\overline{v \sin i} / v_{\mathrm{c}}$ ) is found in the form of "triangular distribution" with a decrease of $p_{*}$ toward very small and very large $v \sin i$ (and $\left.\overline{v \sin i} / v_{\mathrm{c}}\right)$ values. The peak of this distribution is at values $\overline{v \sin i} \approx 200-220 \mathrm{~km} \mathrm{~s}^{-1}$ and $\overline{v \sin i} / v_{\mathrm{c}} \approx 0.41$ $\left(\equiv \overline{v_{\mathrm{t}}} / v_{\mathrm{c}} \approx 0.52\right)$. The decrease of intrinsic polarization toward larger values of projected rotational velocities can be understood in the context of the larger oblateness of CS disks for the stars with a rapid rotation;

8. From the analysis of correlations between different observational parameters we conclude that circumstellar envelopes for the majority of Be stars are optically thin disks with the range of the half-opening angle of $10^{\circ}<\Theta<40^{\circ}$ and the opening angle of CS disks may be smaller for late type stars.

Acknowledgements. I wish to express my sincere thanks to Dr. D. Clarke (Glasgow University) for useful discussions and for the help in the improving of the text. I also thank the referee (Dr. J. Zorec) for his helpful comments. We acknowledge the use of the SIMBAD database and this research has made use of the VizieR Service at Centre de Données Astronomiques de Strasbourg. The research described in this paper was made possible in part by the $99-02-16336$ grant of RFBR.

\section{References}

van den Ancker, M. E., de Winter, D., \& Tjin A Djie, H. R. E. 1998, A\&A, 330, 145

de Araújo, F. X., de Freitas Pacheco, J. A., \& Petrini, D. 1994, MNRAS, 267, 501

Asenijevic, J., Jankov, S., \& Djurasevic, G. 1987, in Proc. IAU Symp. 92, Physics of Be Stars, ed. A. Slettebak, \& T. P. Snow (Cambridge Univ. Press, Cambridge), 200

Ashok, N. M., Bhatt, H. C., Kulkarni, P. V., \& Joshi, S. C. 1984, MNRAS, 211, 471

Ballereau, D., Chauville, J., \& Zorec, J. 1995, A\&AS, 111, 423

Balona, L. A. 1990, MNRAS, 245, 92

Balona, L. A. 1995, MNRAS, 277, 1547

Balona, L. A., Cuypers, J., \& Marang, F. 1992, A\&AS, 92, 533

Bernacca, P. L., \& Perinotto, M. 1970, Contr. Oss. Astron. Asiago, No. 239

Bisnovatyi-Kogan, G. S. 1989, Physical problems of the theory of stellar evolution, Moscow, Nauka, in russian

Bjorkman, J. E., \& Bjorkman, K. S. 1994, ApJ, 436, 818

Bjorkman, K. S., \& Schulte-Ladbeck, R. E. 1994, ASP Conf. Ser., 62, 74

Bjorkman, K. S. 1993, in Proc. IAU Symp. No 162, ed. L. A. Balona, H. F. Henrich, \& J. M. Le Contel, 219

Bjorkman, J. E., \& Cassinelli, J. P. 1993, ApJ, 409, 429

Bjorkman, K. S., Meade, M. R., \& Babler, B. L. 1997, AAS Meeting, 191, 43.03

Briot, D. 1986, A\&A, 163, 67

Brooks, A., Clarke, D., \& McGale, P. A. 1994, Vistas Astron., 38,377

Brown, A. G. A., \& Verschueren, W. 1997, A\&A, 319, 811

Brown, J. C., \& McLean, I. S. 1977, A\&A, 57, 141

Carpenter, K. G., Slettebak, A., \& Sonneborn, G. 1984, ApJ, 286,741

Chandrasekhar, S., \& Münch, G. 1950, ApJ, 111, 142

Clark, J. S., \& Steele, I. A. 2000, A\&AS, 141, 65

Clarke, D., \& Bjorkman, K. S. 1998, A\&A, 331, 1059

Conti, P. S., \& Ebberts, D. 1977, ApJ, 213, 438

Corporon, P., \& Lagrange, A.-M. 1999, A\&AS, 136, 429

Coté, J., \& Waters, L. B. F. M. 1987, A\&A, 176, 93

Coyne, G. V., \& Gehrels, T. 1967, AJ, 72, 887

Cramer, N., Doazan, V., Nicolet, B., et al. 1995, A\&A, 301, 811

Dachs, J., Eichendorf, W., Schleicher, H., et al. 1981, A\&AS, 43,427

Daniel, J.-Y. 1980, A\&A, 86, 198

Doazan, V., Franco, M. L., Stalio, R., \& Thomas, R. N. 1981, in Proc. IAU Symp. No. 98, Be stars, ed. M. Jaschek, \& H.-G. Groth (Dordrecht), 319

Doazan, V., \& Thomas, R. N. 1982, in B Stars with and without Emission Lines, ed. A. Underhill (SP-456, Greenbelt: NASA)

Doazan, V., Malborough, J. M., Morossi, C., et al. 1986, A\&A, 158,1

Doazan, V., Thomas, R. N., \& Bourdonneau, B. 1988, A\&A, 205, L11 
Dolginov, A. Z., Gnedin, Yu. N., \& Silant'ev, N. A. 1995, in Propagation and polarization of radiation in Cosmic Media (Gordon and Breach Publishers)

Dougherty, S. M., Taylor, A. R., \& Clark, T. A. 1991, AJ, 102, 1753

Dougherty, S. M., Waters, L. B. F. M., Burki, G., et al. 1994, A\&A, 290, 609

Elias, II, N. M., Wilson, R. E., Olson, E. C., et al. 1997, ApJ, 484,394

Finkenzeller, U. 1985, A\&A, 151, 340

Fox, G. K. 1991, ApJ, 379, 663

Fox, G. K. 1993a, MNRAS, 260, 513

Fox, G. K. 1993b, MNRAS, 260, 525

Fox, G. K. 1994, ApJ, 435, 372

Fukuda, I. 1982, PASP, 94, 271

Gehrz, R. D., Hackwell, J. A., \& Jones, T. W. 1974, ApJ, 191, 675

Gezari, D. Y., Pitts, P. S., \& Schmitz, M. 1999, CIO5 (vizier.u-strasbg.fr/viz-bin/Cat?II/225)

Ghosh, K. K. 1988, A\&AS, 75, 261

Ghosh, K., Iyengar, K. V. K., Ramsey, B. D., \& Austin, R. A. 1999, AJ, 118, 1061

Grady, C. A., Bjorkman, K. S., Snow, T. P., et al. 1989, ApJ, 339,403

Grillo, F., Sciortiono, S., Micela, G., et al. 1992, ApJS, 81, 795

Halbedel, E. M. 1996, PASP, 108, 833

Hall, J. S., \& Mikesell, A. H. 1953, Publ. US Naval Obs., XVII, Pt.I

Hanuschik, R. W. 1996, A\&A, 308, 170

Heiles, C. 2000, AJ, 119, 923 (vizier.u-strasbg.fr/cgi-bin/VizieR?-source=II/226)

Henrichs, H. F. 1999, in Proc. IAU Coll. No. 169, ed. B. Wolf, O. Stahl, \& A. W. Fullerton (Springer), Lecture Notes in Physics, 523, 305

Herrero, A. 1994, Space Sci. Rev., 66, 137

Hillenbrand, L. A., Strom, S. E., Vrba, F. J., \& Keene, J. 1992, ApJ, 397, 613

Hirata, R. 1993, in Proc. IAU Symp. No 162, ed. L. A. Balona, H. F. Henrich, \& J. M. Le Contel, 448

Hoffleit, D., \& Jaschek, C. 1982, The Bright Star Catalogue, Fourth Revised Edition

Howarth, I. D., Siebert, K. W., Hussain, G. A. J., \& Prinja, R. K. 1997, MNRAS, 284, 265

Huang, L., Hsu, J. C., \& Guo, Z. H. 1989, A\&AS, 78, 431

Hubert-Delplace, A.-M., Jaschek, M., Hubert, H., \& Chambon, M.-T. 1981, in Proc. IAU Symp. No. 98, Be stars, ed. M. Jaschek, \& H.-G. Groth (Dordrecht), 125

Hubert, A.-M., \& Floquet, M. 1998, A\&A, 335, 565

Jaschek, M., \& Egret, D. 1982, in IAU Symp., No. 98, 261 (vizier.u-strasbg.fr/cgi-bin/VizieR?-source=III/67A)

Jaschek, C., \& Jaschek, M. 1993, A\&AS, 97, 807

Jian, S. K., \& Bhatt, H. C. 1995, A\&AS, 111, 399

Jones, T. J. 1979, ApJ, 228, 787

Kaper, L. 1999, in Proc. IAU Coll. No. 169, ed. B. Wolf, O. Stahl, \& A. W. Fullerton (Springer), Lecture Notes in Physics, 523, 193

Leroy, J. L. 1993, A\&AS, 101, 551

Levato, H., \& Maloroda, S. 1970, PASP, 82, 741

Lyubimkov, L. S., Rostopchin, S. I., Roche, P., et al. 1997, MNRAS, 286, 549

Malborough, J. M., Haiqi Chen, \& Waters, L. B. F. M. 1993, ApJ, 208, 650

McDavid, D. 1999, PASP, 111, 494
McDavid, D. 2000, AJ, 119, 352

McLean, I. S., \& Brown, J. C. 1978, A\&A, 69, 291

Mennickent, R. E., Vogt, N., Barrera, L. H., et al. 1994, A\&AS, 106, 427

Moujtahid, A., Zorec, J., Hubert, A. M., Garsia, A., \& Burki, G. 1998, A\&AS, 129, 289

Moujtahid, A., Zorec, J., \& Hubert, A. M. 1999, A\&A, 349, 151

Oudmaijer, R. D., van der Veen, W. E. C. J., Waters, L. B. F. M., et al. 1992, A\&AS, 96, 625

Owocki, S. P., Crenmer, S. R., \& Blondin, J. M. 1994, ApJ, 424,887

Penny, L. R. 1996, ApJ, 463, 737

Poeckert, R., \& Marlborough, J. M. 1976, ApJ, 206, 182

Poeckert, R., Bastien, P., \& Landstreet, J. D. 1979, AJ, 84, 812

Poeckert, R., \& Marlborough, J. M. 1982, ApJ, 252, 196

Porter, J. M. 1996, MNRAS, 280, L31

Porter, J. M. 1997, A\&A, 324, 597

Porter, J. M. 1998, A\&A, 333, 83

Prinja, R. K. 1993, in Proc. IAU Symp. No 162, ed. L. A Balona, H. F. Henrich, \& J. M. Le Contel, 507

Prosser, C. F. 1992, AJ, 103, 488

Quirrenbach, A. 1993, in Proc. IAU Symp. No 162, ed. L. A. Balona, H. F. Henrich, \& J. M. Le Contel, 450

Quirrenbach, A., Bjorkman, K. S., Bjorkman, J. E., et al. 1997, ApJ, 479, 477

Rivinius, Th., Stefl, S., \& Baade, D. 1999, A\&A, 348, 831

Serkowski, K., Mathewson, D. S., \& Ford, V. L. 1975, ApJ 196,261

SIMBAD database, 1999 (simbad.u-strasbg.fr/SIMBAD)

Short, C. I., \& Bolton, C. T. 1994, in Proc. IAU Symp. No. 162 , Pulsation, Rotation and Mass Loss in Early-Type Stars, ed. L. A. Balona, H. F. Henrichs, \& J. M. Le Contel (Kluwer), 171

Slettebak, A., Collins, G. W. II, Boyce, P. B., et al. 1975, ApJS, 29,137

Slettebak, A. 1982, ApJS, 50, 55

Slettebak, A., Wagner, R. M., \& Bertram, R. 1997, PASP, 109, 1

Slettebak, A. 1966, ApJ, 145, 121

Smart, W. M. 1958, Combination of observations (Cambridge Univ. Press)

Snow, T. R. 1981, ApJ, 251, 139

Snow, T. R. 1982, ApJL, 253, L39

Steele, I. A., Negueruela, I., \& Clark, J. S. 1999, A\&AS, 137, 147

Steele, I. A. 1999, A\&A, 343, 237

Sterken, C. 1990, in Proc.: The infrared spectral region of stars, ed. C. Jaschek, \& Y. Andrillat (Cambridge Univ. Press), 319

Straižys, V. 1977, Multicolour stellar photometry (Mokslas Publishers, Vilnius, in russian)

Turner, D. G., Lyons, R. W., \& Bolton, C. T. 1978, PASP, 90, 285

Uesugi, A., \& Fukuda, I. 1982, Revised Catalogue of Stellar Rotational Velocities, Kyoto

van Kerkwijk, M. H., Waters, L. B. F. M., \& Marlborough, J. M. 1995, A\&A, 300, 259

Voshchinnikov, N. V., \& Marchenko, P. E. 1982, Sov. Astron., 59,1115

Waters, L. B. F. M. 1986, A\&A, 162, 121

Waters, L. B. F. M, Coté, J., \& Lamers, H. J. G. L. M. 1987 , A\&A, 185, 206 
Waters, L. B. F. M., \& Marlborough, J. M. 1992, A\&A, 256, 195

Wood, K., Bjorkman, K. S., \& Bjorkman, J. E. 1997, ApJ, 477, 926

Wood, K., Bjorkman, J. E., Whitney, B. A., \& Code, A. D. 1996, ApJ, 461, 828

Wood, K., Bjorkman, J. E., Whitney, B. A., \& Code, A. D. 1996, ApJ, 461, 847

Wolff, S. C., \& Preston, G. W. 1978, ApJS, 37, 371

Wolff, S. C., Edwards, S., \& Preston, G. W. 1982, ApJ, 252, 322

WUPPOL 1999 (www.sal.wisc.edu/WUPPE/polcats/wuppol.html)

Yudin, R. V., \& Evans, A. 1998, A\&AS, 131, 401
Yudin, R. V. 2000, A\&AS, 144, 285

Zorec, J., Mochkovitch, R., \& Garcia, A. 1990, in Proc. Angular Momentum and Mass Loss for Hot Stars, ed. L.A. Wilson, \& R. Stalio (Kluwer Acad. Press), 239

Zorec, J., \& Briot 1997, A\&A, 318, 443

Zorec, J., Fremant, Y., \& Hubert, A. M. 2000a, in The Be Phenomenon in Early-Type Stars, IAU Coll., No. 175, ed. M. Smith, H. Henrich, \& J. Fabregat, ASP Conf. Ser., 214, 330

Zorec, J., Hubert, A. M., \& Moujtahid, A. 2000b, in The Be Phenomenon in Early-Type Stars. IAU Coll., No. 175, ed. M. Smith, H. Henrich, \& J. Fabregat, ASP Conf. Ser., 214, 448

Zorec, J. 2000, private communication 